Louisiana State University

LSU Digital Commons

1973

\title{
Noniterative Integral-Equation Approach to Scattering Problems.
}

Ed Ray Smith

Louisiana State University and Agricultural \& Mechanical College

Follow this and additional works at: https://digitalcommons.Isu.edu/gradschool_disstheses

\section{Recommended Citation}

Smith, Ed Ray, "Noniterative Integral-Equation Approach to Scattering Problems." (1973). LSU Historical Dissertations and Theses. 2500.

https://digitalcommons.Isu.edu/gradschool_disstheses/2500

This Dissertation is brought to you for free and open access by the Graduate School at LSU Digital Commons. It has been accepted for inclusion in LSU Historical Dissertations and Theses by an authorized administrator of LSU Digital Commons. For more information, please contact gradetd@lsu.edu. 


\section{INFORMATION TO USERS}

This material was produced from a microfilm copy of the original document. While the most advenced technological maens to photograph and reproduce this document have been ued, the quality is heavily dependent upon the quality of the original submittud.

The following explanation of techniques is provided to holp you understand markings or patterns which may appear on this reproduction.

1. The sign or "target" for pages apparently lacking from the document photographed is "Missing Pago(s)". If it wes possible to obtain the misaing paro(s) or section, they are spliced into the film along with adjacent pages. This may have necessitated cutting thru an imace and duplicating adjacent pages to insure you complote continuity.

2. When an image on the film is obliterated with a large round black mark, it is an indication that the photographer suspected that the copy may have moved during exposure and thus cause a blurred image. You will find a good image of the page in the adjacent framo.

3. When a map, drawing or chart, etc., was part of the material being photographed the photographer followed a dofinite mothod in "roctioning" the material. It is customary to begin photoing at the upper left hand corner of a large sheet and to continue photoing from beft to right in equal sections with a small overlap. If necessary, sectioning is continued again - beginning below the first row and continuing on until complete.

4. The majority of users indicate that the textual content is of greatest value, however, a somewhat higher quality reproduction could be made from "photographs" if escential to the understanding of the discertation. Silver prints of "photographs" may be ordered at additional charge by writing the Order Department, giving the catalog number, titfe, author and specific paces you wish reproduced.

5. PLEASE NOTE: Some pages may hav indistinct print. Filmed as recoived.

Xerox Univereity Microfilms 300 North Zeeb Rosed Ann Arbor, Michigan 40106 
SMITH, Ed Ray, 1948NONITERATIVE INTEGRAL-EQUATION

APPROACH TO SCATTERING PROBLEMS.

The Louisiana State University and Agricultural and Mechanical College, Ph.D., 1973

Physics, atomic

University Microfilms، A XEROX Company , Ann Ãrbor, Michigan

(C) 1973

ED RAY SMITH

ALL RIGHTS RESERVED 
NONITERATIVE INTEGRAL-EQUATION

APPROACH TO SCATTERING PROBLEMS

\author{
A Dissertation \\ Submitted to the Graduate Faculty of the \\ Loulsiana State University and \\ Agricultural and Mechanical College \\ in partial fulfillment of the \\ requirements for the degree of \\ Doctor of Philosophy \\ in
}

The Department of Physics and Astronomy

by

Ed Ray Smith

B.S., Loulstana State University, 1971

M.S., Jouisiana State University, 1972

August, 1973 


\section{ACKNOWLEDGEMENTS}

The author again wishes to express his sincere appreciation and gratitude to Dr. Ronald J. W. Henry for his invaluable assistance throughout this research project. Gratitude is also extended to Steven P. Rountree and Charles $A$. Weatherford for their many conversations.

Thanks are also due to the LSU Computer Research Center for providing computing facilities and to the U. S. Office of Naval Research* for providing some finaneial support.

${ }^{\star}$ Contract No. N0014-69-A-0211-0004 
This dissertation is dedicated to both my wife and my mother. 


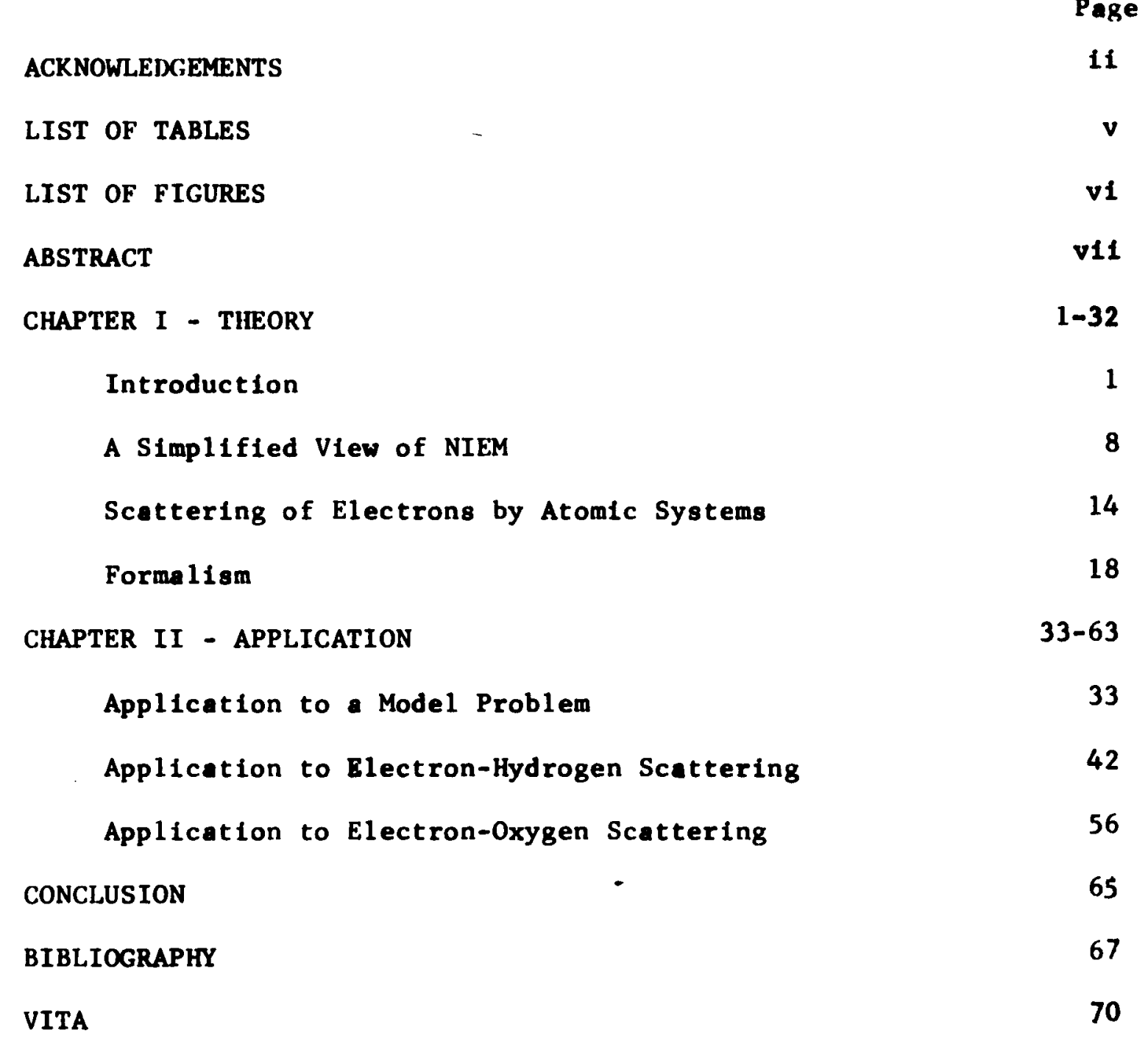


LIST OF TABLES

Page

TABLE

DESCRIPTION

I

Reactance Matrix Elements at Energies Below and

Above Threshold -- (Model Problem).

II

Reactance Matrix Elements at Energies Above the

$n=2$ Threshold -- $(e-H)$.

Partial Wave Cross Sections at Energies Above the

$n=2$ Threshold -- (e-H).

Partial Wave Contributions to the Total Elastic

${ }^{3} \mathrm{P} \rightarrow{ }^{3} \mathrm{P}$ Cross Section Using a 3-State Approximation. 60

V

${ }^{4} \mathrm{P}$ Contributions to the Elastic Cross Section

$\mathrm{Q}\left({ }^{3} \mathrm{P} \rightarrow{ }^{3} \mathrm{P}\right)=(\mathrm{e}-0)$.

61

VI

${ }^{2} \mathrm{P}$ Contributions to the Elastic and Inelastic Cross

Sections - $(e-0)$.

62 


\section{LIST OF FIGURES}

$O\left({ }^{3} P \rightarrow{ }^{3} P\right)$ Cross Section Versus Energy. Solid Curve, 3 State Close-coupling Approximation; Dashed Curve, 4 State Close-coupling Approximation; Circles, Measurements of Sunshine et al. (Ref. 35). 


\section{ABSTRACT}

A procedure which allows for both open and closed channels with exchange, correlation, and orthogonality is formulated for a multichannel scattering process. Noninterative integral equation theory is used to solve the coupled integro-differentiol equations of the scattering problem from zero out to some transformation point where the reactance matrix is projected out to its asymptotic value by using a matricant technique. The method is applied to (1) a two-channel model which has some of the features of a ls - 2p close coupling approximation for e-H scattering, (i1) S-wave scattering of electrons by atomic hydrogen, and (111) P-wave scattering of electrons by atomic oxygen. 


\section{CHAPTER I}

THEORY

\section{Int roduction}

To study the scattering of electrons by atoms, the theoretician has the meens, in principle, to obtain description of the scattering process through the application of the Schrödinger equation. In atomic physics, the quantum-mechenical principles are usually assumed to be understood; however, the technical difficulties encountered in their application to the scattering process is presently overwhelming. In general, the theoretical results disagree with experiment in the energy range below about $100 \mathrm{eV}$, but this range is of greatest interest in describing phenomena that arise, for instance, in the study of plasma physics and stellar atmospheres. The experimentalist, on the other hand, may be able to provide much needed data which cannot be calculated accurately or he may be able to provide accurate measurements on few systems which can be used as check on the theoretical approximetions.

In an attempt to describe phenomena involving electron-atom scattering processes, the theoretician must simplify conceptually the physical picture by assuming a single scattering process in which the electron-atom system is isolated. When the Schrödinger equation is applied to this simplified picture, a description, 
in principle, can be extracted from the total wavefunction for the electron-atom system. For example, total and differentiol crose sections can be calculated from the asymptotic boundary conditions imposed on the total wavefunction. (The boundary conditions at infinity are known, once certain constants referred to as reactance matrix elements are determined numerically. For elastic acatering processes, the reactance matrix is simply tan $\eta_{l}$ where $\eta_{l}$ is the partiol wave phase shift). It 18 apparent that the theoretical description of an electron-stom scattering process reduces to the knowledge of the total wavefunction, but this is where the major technical difficulties arise. For example, even though the potential terms in the Schrödinger equation that describe the electrostatic repulsion between the electrons are not separable (only with respect to the electron coordinates), we make the approximation that the terms in the expansion of the total wavefunction are separable.

For low-energy scattering of electrons by atoms, the best approximation available at this time for obtaining a description of the scattering process 18 by means of the solution of coupled integro-differential equations using numerical techniques. If we expand the total wave function for the system in terms of actual and pseudo-states of the atom and make partial wave expansion for the incident electron, set of coupled integrodifferential enuations result when an appropriate variational principle is applied. Since we cannot solve en Infinite set of 
coupled enuations, the actual atomic atates included in the expansion of the total wave function are restricted to just - few of the lowest lying states; also, small number of pseudo-states are carefully chosen to increase the correlation effects (1.e., the effects of electrostatic reuplsion between 11 the electrons) and polarization effects (1.e., the reaction of the scattered electron back on the atom) that would be neglected by using only a finite number of discrete states. Thus, the infinite set of coupled integro-differential equations are reduced to finite set. This is called a close coupling approximation, the approximation just depending upon the number of atomic states which are retained in the expansion of the total wave function. 1

The addition of preudo-states into the expansion of the total wave function is a more satisfactory approach than using only actual atomic states. With the effects from the pseudostates included in the total wave function, the mathematical description of the scatering process more closely resembles the actual physical description. Thus, the atomic scatering cross sections and related information obtained using this procedure should be of significantly higher auality. However, we must now solve a much larger set of coupled integro-differential equations. It is apperent that the method used in solving the large 
number of coupled equations must be within the storage and time limitations of our present computers. Therefore, with intentions of performing such extensive calculations, we proceeded to investigate four current methods in order to evaluate their speed, storage requirements, simplicity, numerical accuracy, and numerical stability.

The first method (referred to as Numerov) is direct numerical integration of the coupled differential equations using standard computational techniques. 2 The numerical solutions are obtained by integrating the equations outwards and inwards by Numerov's method, with the subsequent matching to obtain a final continuous solution. The asymptotic expansion method of Burke and Schey ${ }^{3}$ is used to determine the reactance matrix. A combination of these methods has been outlined by Smith, et al. 4

Numerov is ressonably well-known; therefore, it provided a good starting point in our investigation. We still use this method, but find that it is a slow technique, when obtaining accurate results, and in some cases it is severely limited by computer storage requirements. The theory involved in understanding the functioning of Numerov is both extensive and technical; however, the accuracy and numerical stabllity 18 generally dependable. Finally, this method can give reliable 
results so that it may be used as a standard against which results from the other three methods may be judged.

Two expansion methode that we investigated were the Herris-Nesbet nethod (HNM) ${ }^{5-7}$ and a combined variable-phaseR-matrix method (VPRMM). 8,9 In these approximate methods, the solution to the coupled equations is given by an expansion in terms of finite basis where the expansion coefficients are to be determined. In their epplication, the majority of the numerical computation required in obtaining the reactance matrix for a given energy reaults from the generation and the diagonalization of Hamiltonian matrices. A main advantage common to both methods is that the matrix diagonalizations only need to be performed once for all energies; thus, a significant amount of computational time can be saved when obtaining reactance matrices at a series of different energy values.

The computational simplicity of these procedures allows for the coupling of more channels than might be attempted in a direct solution such as Numerov; however, the numerical inaccuracies incurred by using amall finite basis may offset this advantage. Moreover, both procedures generally need such a large number of basis functions to obtain consistently accurate results (numerical accuracy at least within $1 \%$ ) 
that the opeed is slower than once hoped, the storage requirenents consequently increase, and the numerical stability can become questionable.

The fourth computational scheme is based on transforming the set of coupled integro-differential equation into set of coupled integral equations. ${ }^{10-18}$ This approach is referred to as a noniterative integral equation method (NIEM). The main advantages of the method are that it is basically simple to understand, very stable and accurate numerically since integrals rather than derivatives are used, as well as fast and free of large storage requirements because of its computational ease. However, when the NIEM is compared with the previous expansion methods, a disadvantage is that a set of integral equations must be solved for each energy value. In addition, an extra equation is obtained for each exchange potential term, as in Numerov, so that a larger set of coupled integral equations must be solved in the NIEM than in the expansion methods. To help in judging the four methods, let us define two categories of methods used for solution of the coupled integrodifferential equations. We have a direct numerical category containing Numerov and the NIEM, and an expansion method category contining the HNM and the VPRMM. Let me emphasize that it is difficult to make a judgment as to the preferred method between the categories because of their distinct approach to the solutions 
of the coupled equations. However, after working with the expansion method category, doubt in the consistent accuracy of the reactance matrix elements turned our attention to the direct numerical category in the hope of finding a method that offered a highly consistent degree of accuracy and stability. After having read the initial publications of Kouri and coworkers on a NIEM, we were favorably Impressed with the apparent advantages of this method, which included not only accuracy and stability, but also numerical speed. At the time, their version of NIEM could only be applied to elastic scattering processes where the exchange potential terms are separable. We decided to extend and modify the method so that it could be applied effectively to the coupled integro-differential equations that arise in the description of electron-atom collisions using a close coupling approximation. Consequently, the goal of this dissertation is to demonstrate the superlor speed, accuracy, and reliability of a new version of the NIEM in comparison with the numerical standard Numerov. Moreover, the NIEM should provide a firm numerical foundation in performing atomic scattering and related calculations where a substantially closer mathematical approximation to reality can be achieved. 
A Simplified View of NIEM

Noniterative integral equation theory as applied to direct potentials 18 well known. 19 Using partial wave decomposition, the radial equation that arises in the scattering of a point particle off a central potential (both having no structure) may be described by the differential equation 20

$$
\left[\frac{d^{2}}{d r^{2}}-\frac{l(l+1)}{r^{2}}+k^{2}\right] F(r)=V(r) F(r)
$$

where $l$ and $k$ are the angular momentum and wave number, $V(r)$ is the direct potential, and $F(x)$ represents the reduced radial part of the incident particle wave function. The associated boundary conditions that $F(r)$ must satisfy are

$$
\begin{aligned}
& F(r) \underset{r \rightarrow 0}{\sim} r^{l+1} \\
& F(r) \underset{r \rightarrow \infty}{\sim} k^{-\frac{3}{2}}\left[\sin \left(k r-\frac{l \pi}{2}\right)+R \cos \left(k r-\frac{l \pi}{2}\right)\right]
\end{aligned}
$$

where $R$ is a constant to be determined (i.e., the reactance matrix). In our analysis we consider an inhomogeneous Strum-Liouville equation 21

$$
\mathcal{L} \cdot(r)=r(r)
$$


in which $\mathcal{L}$ is the self-adjoint differential operator (follows trivially from definition of self-adjoint)

$$
\mathcal{L}=\frac{d^{2}}{d r^{2}}-\frac{l(l+1)}{r^{2}}+k^{2}
$$

and $f(r)$ is the inhomogenuity given by

$$
\mathbf{f}(\mathbf{r})=\mathbf{V}(\mathbf{r}) \mathbf{F}(\mathbf{r})
$$

At this point let us proceed to give the defining properties of a Green's function G:

(1) The interval $0 \leq r<\infty$ is divided into two subintervals by a parameter $t$.

We shall label $G(r)=G_{1}(r)$ for $0 \leq r<t$ and $G(r)=G_{2}(r)$ for $t<r<\infty$.

(2) The functions $G_{1}(r)$ and $G_{2}(r)$ each satisfy the homogeneous Sturm-Liouville equation: that 18 ,

$$
\begin{array}{ll}
\mathscr{L}_{G_{1}}(r)=0 & 0 \leq r<t, \\
\mathscr{L}_{G_{2}}(r)=0 & t<r<\infty .
\end{array}
$$

(3) At $r=0, G_{1}(r)$ satisfies the boundary conditions we impose on $F(r)$. Correspondingly, ss $r$ tends to infinity, $G_{2}(r)$ satisfies the boundary conditions imposed on $F(r)$. 
(4) We demand that $G(r)$ be continuous at $r=t$ (continuity requirement)

$G_{1}(t)=G_{2}(t)$

(5) We require that $G^{\prime}(r)$ be discontinuous, specifically that (jump condition)

$\left.\frac{d}{d r} \quad G_{2}(r)\right|_{t}-\left.\frac{d}{d r} G_{1}(r)\right|_{t}=1$ 2.6

These properties (requirements) make the Green's function a function of two variables, $G(r, t)$. Furthermore, we note that $G(r, t)$ depends both on the form of the differential operator $\mathcal{X}$ and on the boundary conditions that $F(r)$ must satisfy. With these notes in mind, let us proceed to construct the the Green's function.

We ma introduce the single variable auxillary functions $G^{(1)}$ and $G^{(2)}$ which are obtained by letting

$$
\begin{aligned}
& G^{(1)}(k r)=k^{\frac{1}{2}} r j_{\ell}(k r) \\
& G^{(2)}(k r)=k^{\frac{1}{2}} r y_{\ell}(k r)
\end{aligned}
$$


where $j_{\ell}(x)$ and $y_{\ell}(x)$ are spherical Bessel and Neumann functions. We see that $G^{(1)}(\mathrm{kr})$ and $G^{(2)}(\mathrm{kr})$ both satisfy the homogeneous Sturm-Liouville equation; only $G^{(1)}(k r)$ satisfies the boundary condition on $F(r)$ at $r=0$ and both functions help satisfy the boundary condition as $r$ tends to infinity:

$$
\begin{aligned}
& G^{(\alpha)}(k r) \underset{r \rightarrow 0}{\sim}\left\{r^{-l}, \alpha=2\right. \\
& G^{(\alpha)}(k r) \underset{r \rightarrow \infty}{-}\left\{k_{-k^{-\frac{1}{2}} \cos \left(k r-\frac{l \pi}{2}\right), \alpha=2 .}^{k^{-\frac{l}{2}} \sin \left(k r-\frac{\ell \pi}{2}\right), \alpha=1}\right.
\end{aligned}
$$

We may now take

$$
G(r, t)= \begin{cases}C_{1} G{ }^{(1)}(k r) & 0 \leqslant r<t \\ C_{2} G(2)(k r) & t<r<\infty .\end{cases}
$$

To identify $\mathrm{C}_{1}$ and $\mathrm{C}_{2}$ let us continue to apply the conditions on $G(r, t)$. Continuity at $r=t$ requires

$$
c_{1} j_{\ell}(k t)=c_{2} y_{\ell}(k t) .
$$


Next, the jump disconinuity at $r=t$ implies

$$
\left.C_{2} G^{\prime(2)}(k r)\right|_{t}-c_{1} G^{(1)}(k r) !=1
$$

where the prime denotes differentiation with respect to $r$. Finally, the Wronskian relation between $G^{(1)}(k r)$ and $G^{(2)}(k r)$ is

$$
G^{(1)}(k r) G^{\prime(2)}(k r)-G^{(2)}(k r) G^{\prime(1)}(k r)=1 .
$$

We can now Identify $C_{1}$ and $C_{2}$ using Equations (2.10), (2.11) and (2.12):

$$
\begin{aligned}
& C_{1}=G^{(2)}(k t) \\
& C_{2}=G^{(1)}(k t) .
\end{aligned}
$$

Thus, the Green's function is

$$
G(r, t)= \begin{cases}G^{(1)}(k r) G^{(2)}(k t), & 0 \leq r<t \\ G^{(1)}(k t) G^{(2)}(k r), & t<r<\infty .\end{cases}
$$

The differential Equation (2.1) and its associated boundary conditions Equation (2.2) are then transformed into an integral equation, in the usual way,

$$
F(r)=\int_{0}^{\infty} G(r, t) f(t) d t+G^{(1)}(k r)
$$


where the first term on the r.h.s. Is the inhomogeneous part of the integral equation and the second term is a homogeneous solution of Equation (2.1) that is finite for all values of $r$. 
Scattering of Electrons by Atomic Systems

A theory of scattering of electrons by atoms or lons with any number of incomplete subshells has been developed within a close-coupling approximation by Smith and Morgan. 22 Allowance is made for the target system to be excited to an electronic configuration constructed using the same core (discrete) orbitals for all electronic configurations. The radial part of the one-electron orbitals for a discrete subshell are assumed known and Identical for each electron in given discrete oubshell; the scattering (continuum) functions are given as the solution of coupled integro-differential equations with prescribed boundary conditions. The form of these equation is such that the continuum functions are orthogonal to all the discrete orbitals. Moreover, the potential terms appearing in the equations are written in terms of generalized angular momentum recoupling coefficients. 23

We will expand the total wave function for the system, atom plus incident electron, in terms of a completely ant1symmetric expansion involving core wave functions and free electron orbitals using a close-coupling approximation:

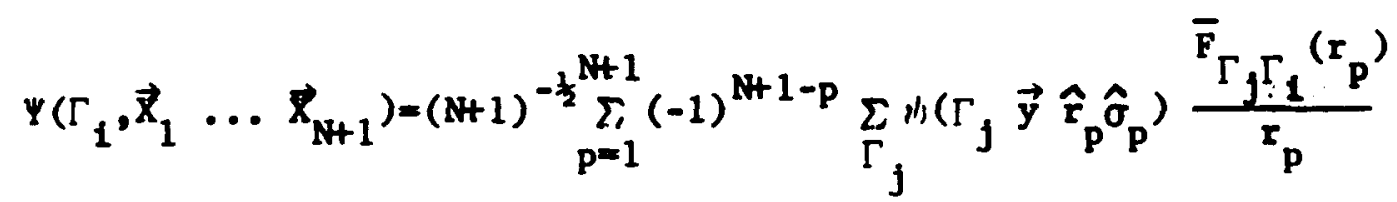

where $N$ is the total number of electrons in the atom, $\Gamma_{1}$ is a complete set of quantum numbers required to specify the system 
in an initial state $1, \vec{X}_{1}$ denotes both the spin $(\vec{\sigma})$ and spatial coordinates $(\vec{r})$ of the $f^{\text {th }}$ electron, $\vec{y}$ denotes the spin and spatial coordinates of $a 11 N+1$ electrons except the $p^{\text {th }}$ electron, (I) denotes the core wave functions and $\bar{F}$ denotes the continuum orbitals.

When applying the appropriate variational principle with the total wave function, the analysis of the exchange potential terms can be greatly simplified if we constrain the continuum functions to be orthogonal to all the discrete orbitals. However, the constraint can be thought of as only preventing electron capture. 22 To place the possibility of electron capture (speaking of only momentary capture) back into the trial wave function, we must add to Equation (3.1) wave functions that contain electron capture configurations:

$$
\begin{aligned}
\Psi\left(\Gamma_{1}, x_{1} \ldots x_{N+1}\right)=(N+1)^{-\frac{1}{2}} \sum_{p=1}^{N+1}(-1)^{N+1-p} \sum_{\Gamma_{j}} \psi\left(\Gamma_{j}+\hat{r}_{p} \hat{\partial}_{p}\right) \frac{F_{1} \Gamma_{1}\left(r_{p}\right)}{r_{p}} \\
+\sum_{\mu} C_{\mu}^{\Gamma_{1}} \Phi_{\mu}\left(L S_{\pi}, x_{1} \ldots x_{N+1}\right)
\end{aligned}
$$

where $F_{\Gamma_{j} \Gamma_{1}}(r) \equiv F_{j 1}(r)$ is orthogonal to all discrete orbitals, $\Phi_{\mu}$ denotes an explicit electron capture wave function, $L$ and $S$ are the total orbital and spin angular momenta, $\pi$ is the parity, 
and $C_{\mu}^{1}$ are variationa1ly determined coefficients that add back into the trial wave function the effect of electron capture.

The variational principle that we employ is

$$
\delta\left[L_{1 j}-R_{1 j}\right]=0
$$

where $R_{1 j}$ are the elements of the reactance matrix defined in terms of the asymptotic form of $F_{1 j}$ in the open channels $\left(k_{1}^{2}>0\right)$

$$
F_{1 j}(r) \underset{r \neq \infty}{\sim} k_{1}^{-\frac{1}{2}}\left[\delta_{1 j} \sin \theta_{1}+R_{1 j} \cos \theta_{1}\right]
$$

where

$$
\theta_{1} \equiv k_{1} r-\ell_{1} \frac{\pi}{2}
$$

The matrix $L$ is defined by

$$
L_{1 j} \equiv \int \Psi\left(\Gamma_{1}, \vec{X}_{1} \ldots \vec{x}_{N+1}\right)\left[H_{N+1}-E\right] \Psi\left(\Gamma_{j}, \vec{x}_{1} \ldots \vec{x}_{N+1}\right) d \vec{x}_{1} \ldots d \vec{x}_{N+1}
$$

where $E$ is the total energy (atom plus incident electron), and $\mathrm{H}_{\mathrm{NH}}$ is the total Hamiltonian. The $\delta$ in Equation (3.3) Implies variations with respect to both $F_{i j}$ and $c_{\mu}^{i}$. In other words, the bracketed term in Equation (3.3) is stationery with respect to variations 


$$
\delta F_{1 j}(r) \underset{r \rightarrow \infty}{-} k_{1}^{-\frac{1}{2}} \delta R_{1 j} \cos \theta_{i}
$$

and arbitrary $\delta C_{\mu}^{1}$. Moreover, the variation with respect to $F_{1 J}$ is still subject to the requirement that it is orthogonal to all discrete orbitals. We introduce undetermined Lagrange multipliers $M$ to enforce this constraint on $F_{1 j}$. The mathematical form of the resulting coupled integro-differential equations for the continuum function $F_{i j}$ is stated in the next eection. 


\section{Formal1sm}

In this section we present the NIEM as applied to multichannel scattering problem. The equations that arise in the close-coupling formalism 4 of an electron-complex atom acattering problem may be described by the linear coupled integro-differential equations

$$
\begin{gathered}
{\left[\frac{d^{2}}{d r^{2}}-\frac{l_{1}\left(l_{1}+1\right)}{r^{2}}+k_{1}^{2} F_{1 j}(r)=\sum_{n}\left[v_{i n}(r)+w_{i n}(r)+z_{i n}(r) F_{n j}(r)\right.\right.} \\
+\sum_{\lambda}^{\sum} \delta_{l_{1} l_{\lambda}} M_{\lambda}^{1 j} P_{\lambda}(r) \quad 1, j=1,2 \ldots N
\end{gathered}
$$

where $N$ is the number of channels, $\ell_{1}$ and $k_{1}$ are the angular momentum and wave number for channel $1, \underline{V}$ is the direct potential matrix, the integral operators $\underline{W}$ and $\underline{Z}$ are the exchange and correlation potential operators, and $P_{\lambda}(r)$ is the reduced radial part of the atomic electron orbital associated with the label $\lambda$. The $\underline{M}$ are the undetermined Lagrange multipliers that insure that the solution satisfies its required constraints, $1 . e$, the incident electron wave function $\underline{F}(\vec{r})$ is orthogonal to all the bound atates $\lambda$ of the atom. F is a square matrix, which represents the reduced radial part of the incident electron wave function, with $j$ denoting the incident channel and 1 the outgoing channel. We allow for the possibility that a channel can be open $\left(k_{1}^{2}>0\right)$ or closed $\left(k_{1}^{2}<0\right)$. 
The exchange potential for this problem 18 defined by

$$
\sum_{n} W_{1 n}(r) F_{n j}(r)=\underset{\alpha_{1}}{\Sigma^{\prime}} B_{1}^{\left(\alpha_{1}\right)} Y_{t}\left(P_{\rho_{1}}, F_{n j} ; r\right) P_{\rho_{n}}(r)
$$

with

$$
Y_{t}(A, B ; r)=\frac{1}{r^{t+1}} \int_{0}^{r} A(x) B(x) x^{t} d x+r^{t} \int_{r}^{\infty} \frac{A(x) B(x)}{x^{t+1}} d x
$$

where $\left|\ell_{\rho_{1}}-\ell_{n}\right| \leq t \leq \ell_{\rho_{1}}+\ell_{n}$

$$
\left|\ell_{\rho_{n}}-\ell_{1}\right| \leq t \leq \ell_{\rho_{n}}+\ell_{1}
$$

and $\ell_{\rho_{1}} 18$ the angular momentum associated with the atomic state $\rho_{1}$. The prime on the summation in Equation (4.2) denotes a sum over only exchange terms and, thus, the composite index is defined as $\alpha_{1}=\left(t, i, n, \rho_{1}, \rho_{n}\right)$, and $B_{1}^{\left(\alpha_{1}\right)}$ are the exchange potential coefficients. The exchange terms cited here are not separable and, therefore, require a different treatment from that give by Sams and Kour $1.10-13$

The correlation potential for this scattering problem can be described, in general, by the expression

$$
\left.\sum_{n} z_{\sum_{n}}\right) F_{n j}(r)=\sum_{n} \underset{\zeta=1}{\sum V}\left[\sum_{v=1}^{N V} E_{\zeta}^{U} v_{v i}(r)\right] \int_{0}^{\infty} v_{\zeta n}(x) F_{n j}(x) d x
$$


where NV is the number of correlation terma in a channel, $E_{j}^{U}$ are correlation constants, and $V_{u i}(r)$ are correlation potential elements.

The integral equation for $F_{i j}(r)$ is

$$
\begin{aligned}
& F_{1 j}(r)-\Delta_{i} \delta_{1 j} G_{i}^{(1)}\left(k_{1} r\right)+G_{i}^{(2)}\left(k_{i} r\right) \int_{0}^{r} G_{1}^{(1)}\left(k_{1} x\right) S_{1 j}(x) d x \\
& -G_{1}^{(1)}\left(k_{1} r\right) \int_{0}^{r} G_{i}^{(2)}\left(k_{1} x\right) S_{i j}(x) d x+G_{1}^{(1)}\left(k_{1} r\right) \\
& X \int_{0}^{\infty} G_{1}^{(2)}\left(k_{1} x\right) S_{i j}(x) d x
\end{aligned}
$$

with

$$
\Delta_{1}= \begin{cases}1 & 1 \text { open } \\ 0 & 1 \text { closed }\end{cases}
$$

where $\underline{S}$ is the source term on the r.h.s. of Equation (4.1), $G_{i}^{(\alpha)}\left(k_{1} r\right)$ are the appropriate Greer's functions that are defined as real diagonal matrices 24

$$
G_{i}^{(1)}\left(k_{1} r\right)= \begin{cases}k_{1}^{\frac{1}{2}} r j_{\ell_{1}}\left(k_{1} r\right) & \text { i open } \\ \left(2 x_{i}\right)^{\frac{1}{2}} r e_{\ell_{1}}\left(x_{1} r\right) & \text { i closed }\end{cases}
$$




$$
G_{1}^{(2)}\left(k_{1} r\right)= \begin{cases}k_{1}^{\frac{3}{2}} r y_{\ell_{1}}\left(k_{1} r\right) & 1 \text { open } \\ \frac{-\left(2 k_{1}\right)^{\frac{1}{2}}}{\pi} r b_{\ell_{1}}\left(k_{1} r\right) & 1 \text { closed }\end{cases}
$$

where $J_{\ell}(x)$ and $y_{\ell}(x)$ are spherical Bessel and Neumann functions, $a_{l}(x)$ and $b_{\ell}(x)$ are appropriately defined modified spherical Bessel and Neumann functions, and the closed channel modified wave number is $x_{1}=\left|k_{1}\right|$. The boundary conditions associated with the scattering problem are satisfled with the following Green's functions

$$
\begin{aligned}
& G_{i}^{(1)}\left(k_{1} r\right) \underset{r \rightarrow 0}{e_{1}^{l_{1}+1}} \\
& G_{i}^{(1)}\left(k_{1} r\right) \underset{r \rightarrow \infty}{\left(2 x_{1}\right)^{-\frac{3}{2}} e^{x_{1} r}} \quad \begin{array}{ll}
k_{1}^{-\frac{3}{2}} \sin \left(k_{1} r-l_{1} \frac{\pi}{2}\right) & 1 \text { open } \\
& \text { i closed }
\end{array} \\
& G_{i}^{(2)}\left(k_{1} r\right) \underset{r \rightarrow \infty}{\sim} \begin{cases}-k_{i}^{-\frac{1}{2}} \cos \left(k_{1} r-l_{i} \frac{\pi}{2}\right) & 1 \text { open } \\
-\left(2 x_{i}\right)^{-\frac{1}{2}} e^{-x_{1} r} & 1 \text { closed. }\end{cases}
\end{aligned}
$$

Furthermore, note that the index $j$ in Equation (4.3) not only denotes incident channel but also the different independent solutions corresponding to channel 1 . 
Another solution to Equation (4.1) is defined by neglecting the normalization term as in variable phase theory 9

$$
\begin{aligned}
& d_{i j}(r)=G_{i}^{(1)}\left(k_{1} r\right) H_{i j}^{(2)}(r)-G_{1}^{(2)}\left(k_{1} r\right) H_{i f}^{(1)}(r) \\
& H_{i j}^{(2)}(r)=\delta_{1 j}-\int_{0}^{r} G_{1}^{(2)}\left(k_{1} x\right) s_{i j}(x) d x \\
& H_{i j}^{(1)}(r)=-\int_{0}^{r} G_{i}^{(1)}\left(k_{1} x\right) s_{i j}(x) d x
\end{aligned}
$$

In contrast, Sams and Kouri ${ }^{10}$ retain the normalization term. If al1 the channels are open, the asymptotic boundary conditions on 12 and $\underline{F}$ are

$$
\begin{aligned}
& h_{1 j}(r) \underset{r \rightarrow \infty}{=} k_{i}^{-\frac{1}{2}}\left[A_{i j} \ln \left(k_{1} r-l_{i} \frac{\pi}{2}\right)+B_{1 j} \cos \left(k_{1} r-l_{1} \frac{\pi}{2}\right)\right] \\
& F_{i j}(r) \underset{r \rightarrow \infty}{=} k_{1}^{-\frac{3}{2}}\left[\delta_{1 j} \ln \left(k_{1} r-l_{1} \frac{\pi}{2}\right)+R_{1 j} \cos \left(k_{1} r-l_{i} \frac{\pi}{2}\right],\right.
\end{aligned}
$$

where $A_{i j}$ and $B_{i j}$ are the asymptotic 1 imits of the functions $H_{1 j}^{(2)}(r)$ and $\mathrm{H}_{1 j}^{(1)}(r)$, respectively, and $R_{i j}$ is the reactance matrix. As noted previously, the only difference between $\Psi(r)$ and $\underline{F}(r)$ 1s their respective normalizations. Since the normalization for this problem is chosen by requiring the solution of Equation (4.1) to satisfy the asymptotic boundary conditions given in Equation (4.8b), all we need do is asymptotlcally renormallze $k(r)$ to correapond lo liqual ton (4. Hl,), 
and appropriately choose the resulting reactance matrix. Renormalizing W2(r), we obtain the linear combination

$$
\Phi_{i j}(r)=\sum_{n} \psi_{i n}(r) A_{n j}^{-1}
$$

If the linear coupled integro-differential equation for $h(r)$ are transformed by Equation (4.9), we observe that $\Phi(r)$ is also a solution of the linear equations where the undetermined Lagrange multipliers, in this case, insure that $\Phi(\vec{r})$ is orthogonal to all discrete orbitals. Noticing that $\Phi$ and $\underline{E}$ both satisfy Equation (4.1) and also satisfy the same boundary conditions, we can conclude that they are identical by uniqueness. Therefore, the reactance matrix elements $R_{1 j}$ can be determined from knowledge of the asymtotic behavior of the unnormalized function w from the equation

$$
R_{i j}=\sum_{n} B_{i n} A_{n j}^{-1}
$$

Next, if we also allow some of the channels to be closed, the procedure for obtaining the reactance matrix elements $R_{1 j}$ is somewhat more complicated. Unlike Sams and Kouri, ${ }^{12}$ we do not explicitly solve open and closed channel equations; rather, we obtain the reactance matrix by numerically forcing the appropriate closed and open channel asymptotic boundary conditions. Notice 
that $\delta:$ a closed channel 1 , the unnormalized solution $\psi_{1 j}(r)$ exponentially grows asymptotically, while the solution $F_{i j}(r)$ exponentially decays asymptotically. From the structure of Equation ( 4.7$)$, we see that a column vector of $12(r)$ depends only on the elements in that column. In order to have a well-behaved solution $/_{1 j}(r)$ for channels 1 and $j$ both open, it 18 evident that we must somehow numerically force $d_{\operatorname{mn}}(r)$ to exponentially decay asymptotically for channel m closed and channel $n$ open. In particular, consider a two-channel problem with channel $i=1$ open and channel $i=2$ closed. If we transform $\mho_{21}(r)$ to zero using upper triangularization 25 at - sufficiently large enough value of $r$, where $F_{21}(r)$ would also be zero, then $\pi_{11}(r)$ would be well-behaved beyond that point. (The matrix 这 denotes that the matrix \& has been transformed appropriately 80 that $\widetilde{\psi}_{21}$ is zero aymptotically and also a solution of Equation (4.1)). The transformation is aimilar to that of Equation (4.9). Finally, if the open channel asymptotic boundary conditions are now forced on $\Psi_{11}(r)$, we can obtain the reactance matrix element in the open channel as desired.

The unnormalized integral equation stated in Equation (4.7) may be rewritten as

$$
\begin{aligned}
\|_{i j}(r) & =\delta_{i j} G_{i}^{(1)}\left(k_{1} r\right)+\int_{0}^{r} G_{i}^{(2,1)}(r \mid x)\left[\sum_{n}\left(v_{i n}(x)+W_{i n}(x)+Z_{i n}(x) y\right) \psi_{n j}(x)\right. \\
& \left.+\frac{\Sigma_{\lambda} \ell_{i} \ell_{\lambda}}{M^{i j}} P_{\lambda}(x)\right] d x
\end{aligned}
$$


with

$$
G_{i}^{(2,1)}(r \mid x)=G_{i}^{(2)}\left(k_{i} r\right) G_{i}^{(1)}\left(k_{1} x\right)-G_{1}^{(1)}\left(k_{1} r\right) G_{i}^{(2)}\left(k_{1} x\right) .
$$

Since the exchange potential terms are not separable, they can be rewritten so that Fquation $(4.11)$ becomes

$$
\begin{aligned}
& \Downarrow_{1 j}(r)=\delta_{1 j} G_{1}^{(1)}\left(k_{1} r\right)+\int_{0}^{r} G_{1}^{(2,1)}(r \mid x)\left[\sum_{n} v_{1 n}(x) \psi_{n j}(x)\right. \\
& +\Sigma_{\alpha_{1}}^{B_{1}^{\left(\alpha_{1}\right)}}{ }_{P_{\rho_{n}}}(x)\left\{\frac{1}{x^{t+1}} \int_{0}^{x} P_{\rho_{1}}(y) \|_{n j}(y) y^{t} d y-x \int_{0}^{t} \int^{x} \frac{{ }_{n_{1}}(y) d_{n j}(y)}{y^{t+1}} d y\right\} \\
& +\sum_{n} z_{i n}(x) \mho_{n j}(x)+\sum_{\lambda} \delta_{\ell_{1} \ell_{\lambda}}^{M^{i j} P_{\lambda}(x)} \\
& \left.+\sum_{n_{1}} B_{1}^{\left(N_{1}\right)}{ }_{P_{\rho_{n}}}(x) x^{t} \int_{0}^{\infty} \frac{P_{\rho_{1}}(y) b_{n j}(y)}{y^{t+1}} d y\right] d x \text {. }
\end{aligned}
$$

The solution to Equation (4.11) can be written as

$$
\psi_{1 j}(r)=\psi_{1 j}^{(0)}(r)+\Sigma_{m \alpha_{m}} \psi_{1 m}^{\left(\alpha_{m}\right)}(r) c_{m j}^{\left(\alpha_{m}\right)}
$$

with 


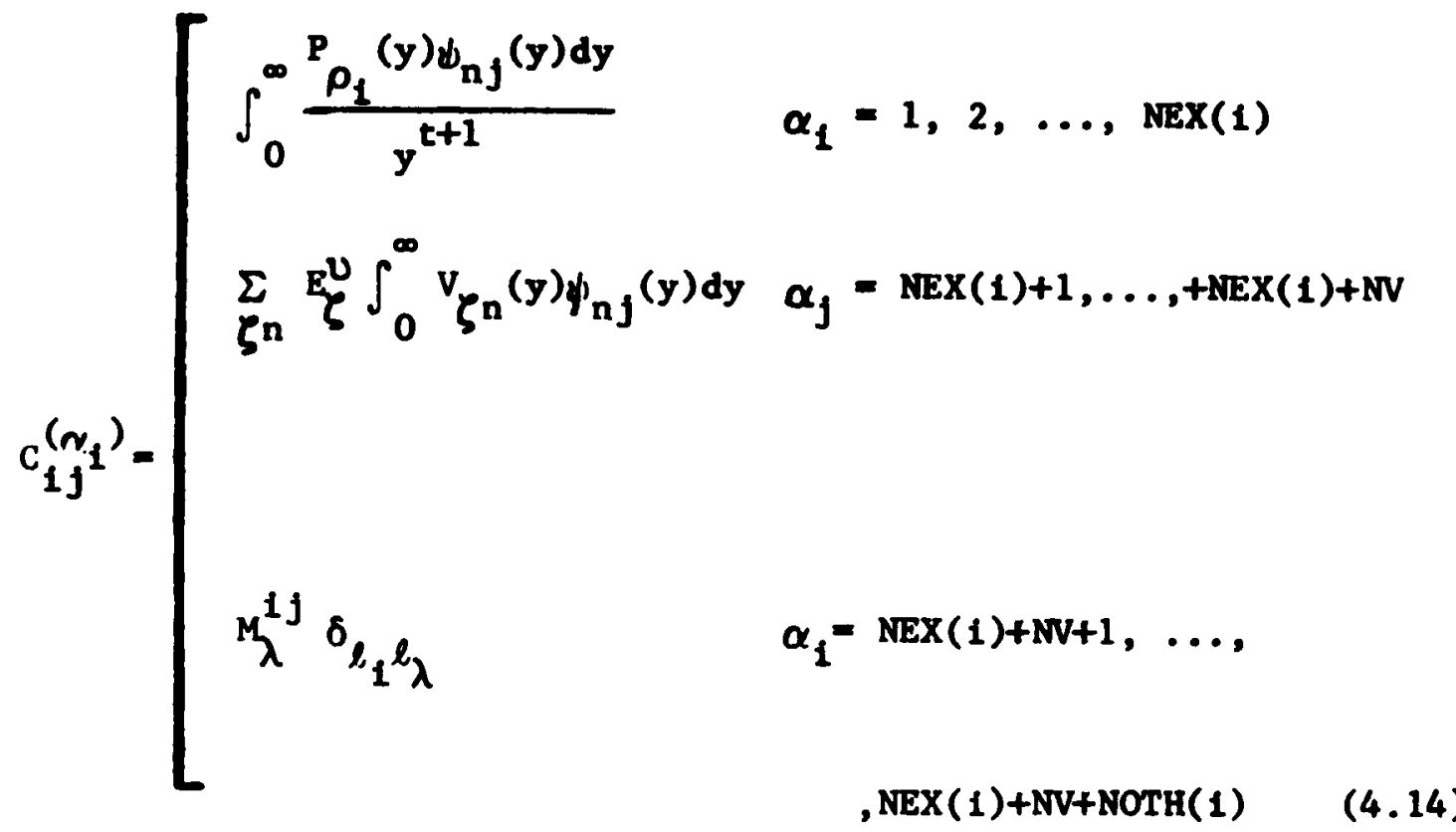

$$
\begin{aligned}
& \psi_{1 \mathrm{~m}}^{(N)}(x)=\delta_{\alpha, 0}{ }^{5} \mathrm{Im}_{1}^{(1)}\left(k_{1} r\right)+\int_{0}^{r} G_{1}^{(2,1)}(r \mid x)\left[\sum_{n} v_{1 \mathrm{n}}(x) \psi_{n m}^{(\alpha)}(x)\right.
\end{aligned}
$$

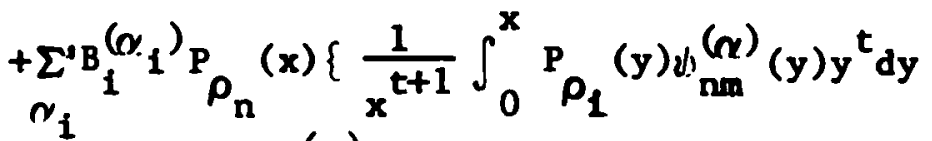

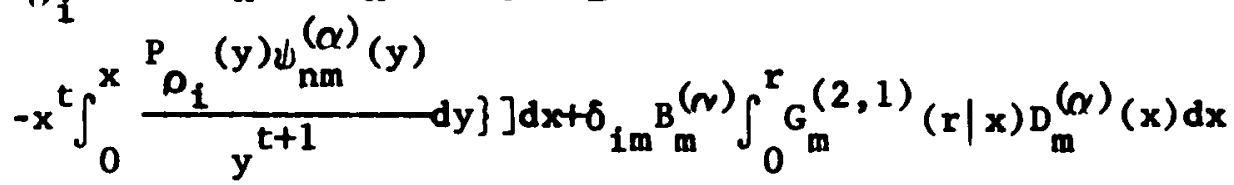

and

$$
B_{1}^{(\alpha)}= \begin{cases}0 & \alpha=0 \\ B_{1}^{\left(N_{1}\right)} & \alpha=\alpha_{1}=1,2, \ldots, \operatorname{NEx}(1) \\ 1 & \text { otherwise }\end{cases}
$$




$$
D_{1}^{(x)}=\left\{\begin{array}{cl}
0 & \alpha=0 \\
P_{\rho_{n}}(x) x^{t} & \alpha=\alpha_{1}=1,2, \ldots, \operatorname{NEX}(1) \\
V_{v 1}(x) & \alpha=\alpha_{1}=\operatorname{NEX}(1)+1, \ldots, \operatorname{NEX}(1)+N V \\
P_{\lambda}(x) & \alpha=\alpha_{1}=\operatorname{NEX}(1)+N V+1, \ldots, \operatorname{NEX}(1)+N V+N O T H(1)
\end{array}\right.
$$

where NEX(1) is the number of exchange terms in channel 1 , and NOTH(1) is the number of orthogonality terms. For a given pair $\left(m, n_{m}\right)$, we can generate a column vector $\left.\psi_{1 m}^{(\alpha)}\right)^{\prime}$ using Equation (4.15). Similarly, another column vector $\psi_{1 \mathrm{~m}}^{\left(\alpha^{\prime}\right)}$ can be generated for a given peir $\left(m, \alpha_{m}^{\prime}\right)$. If $D_{m}\left(\alpha_{m}\right)=D_{m}\left(\alpha_{m}^{\prime}\right)$, then the corresponding column vectors are related by

$$
\psi_{m}^{\left(\alpha_{m}\right)}=\frac{B_{m}^{\left(\alpha_{m}\right)}}{{ }_{B_{m}}^{\left(\alpha_{m}^{\prime}\right)} \psi_{m}}\left(\alpha_{m}^{\prime}\right)
$$

In addition, computation time can be saved by making the observation that the auxiliary function $\psi^{(\alpha)}$ can be evaluated without any matrix inversion. 10

The constraint condition that determines the constante $x^{1 j}$ is

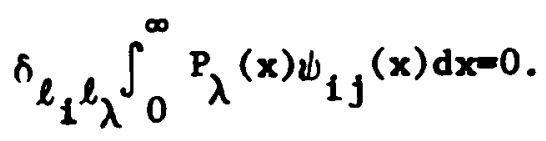

We impose the constralnt that the radial functlun must. be orthop:()inl to all the atomic orbitals. We further introduce the quentily 


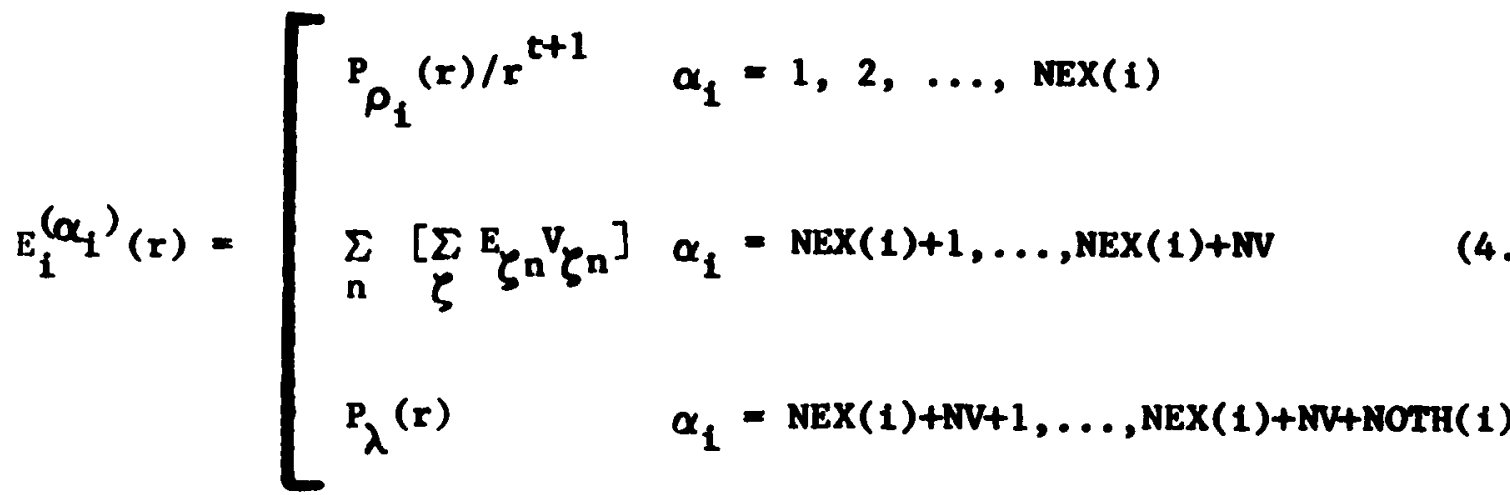

so that Equation (4.18) and Equation (4.14) may be combined to yield

$$
A_{\alpha_{1}} c_{i j}^{\left(\alpha_{1}\right)}=\int_{0}^{\infty} E_{i}^{\left(\alpha_{1}\right)}(y) \psi_{n j}(y) d y
$$

with

$$
\phi_{\alpha_{1}}= \begin{cases}1 & \alpha_{1}=1,2, \ldots, \operatorname{NEX}(1)+N V \\ 0 & \text { otherwise. }\end{cases}
$$

Substituting Enuation (4.13) Into Equation (4.19) yields a set of simultaneous equations to be solved for the constants $c_{1 j}\left(\alpha_{1}\right)$, namely

$$
\phi_{i} c_{1 j}^{\left(\alpha_{1}\right)}=D_{i j}^{\left(\alpha_{1}, 0\right)}+\sum_{m \beta_{m}} c \xi_{m j}\left(\varphi_{m}\right) D_{1 m}^{\left(\alpha_{1}, \beta_{m}\right)}
$$

with

$$
D_{i j}^{\left(N_{1}, \beta\right)}=\int_{0}^{\infty} E_{1}^{\left(N_{1}\right)}(y) d_{n j}(\beta)(y) d y, \beta=0, \beta_{j} .
$$


Noting that the exchange correlation and orthogonality terms exhibit the short-ranged behavior of the atomic electron orbitals, we conclude that the upper 1 imit of the integral in Equation (4.19) is basically the range of these orbitals.

The unnormalized solution $\underline{W}$ can be determined at a transformation point $r_{t}$ by integrating the homogeneous solution $\psi^{(0)}$ and the particular solutions $\psi^{(\alpha)}$ of Equation (4.15) from zero to $r_{t}$. Th1s value of $r_{t}$ is the point at which the integrals involving exchange, correlation and orthogonality terms have converged. Once the auxiliary functions are obtalned, the constants $D_{1 j}^{\left(\alpha_{1}, \beta\right)}$ are simply determined from Equation (4.21). Then, the constants $\mathrm{C}_{1 j}^{\left(\alpha_{1}\right)}$ are computed by matrix inversion from Equation (4.20). Hence, the solution $\underset{12}{ }\left(r_{t}\right)$ may be determined from Equation (4.13); only at this stage is it first possible to force the closed and open channel boundary conditions on 1 . Note that any further contribution to $U_{2}(r)$ for $r>r_{t}$ comes only from the direct potential $\underline{v}(r)$. Knowing $\underline{y}^{\prime}\left(r_{t}\right), \underline{H}^{(1)}\left(r_{t}\right)$, and $\underline{H}^{(2)}\left(r_{t}\right)$, we may elther continue integrating $\psi(r)$ to a point where the potential vanishes, or we may "project" the $\underline{H}$ matrices to their asymptotic value by a technique given below. Finally, we obtaln the reactance matrix from Equation (4.10).

The projection procedure stems from noting that 
30

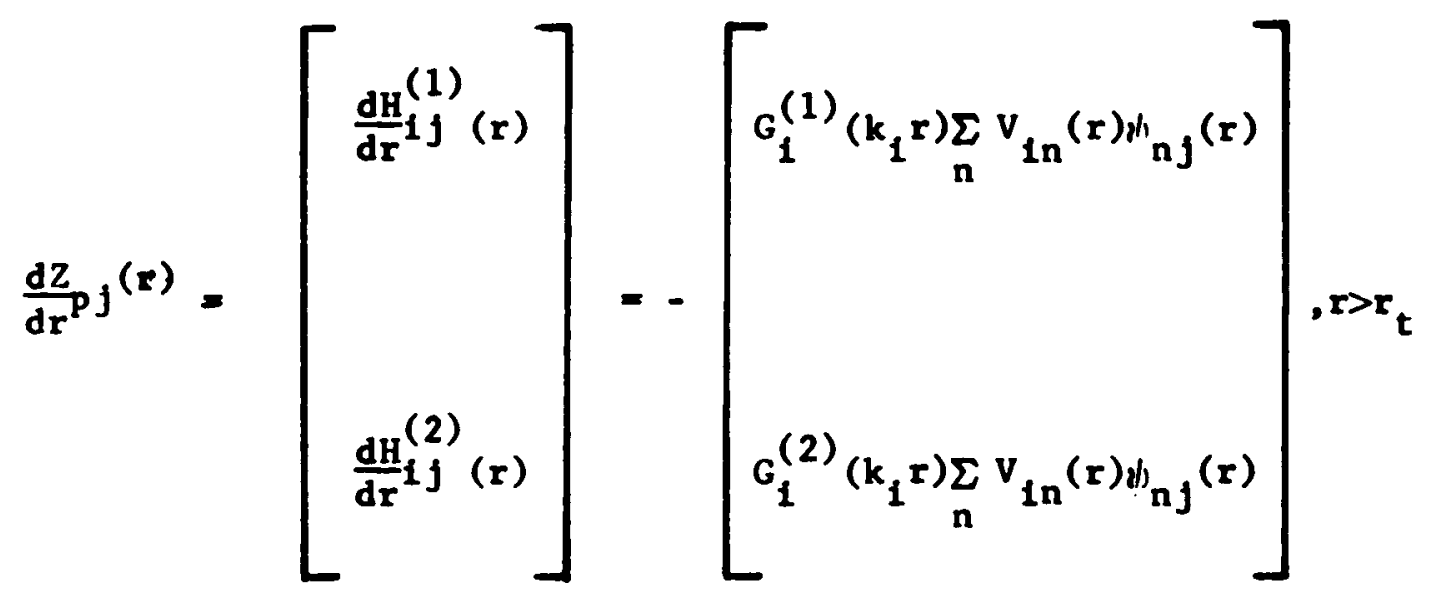

where $p=1,2, \ldots, 2 \mathrm{~N}$

Substituting Equation (4.7) into Equation (4.22) yields

$$
\begin{aligned}
& \frac{d Z}{d r^{p j}}(r)=\sum_{q=1}^{2 N} M_{p q}(r) Z_{q j}(r), \\
& M_{p q}(r)=G \frac{\left(\alpha_{p}\right)}{p}\left(k_{\bar{p}} r\right) G \underset{q}{\left(\beta_{q}\right)}\left(k_{-q} r\right) V_{\overline{p q}}(r)(-1) \beta_{q}
\end{aligned}
$$

with

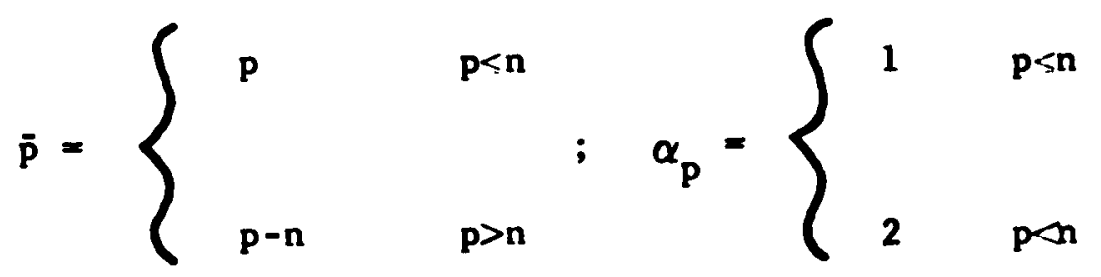

$$
\vec{q}=\left\{\begin{array}{lll}
q & q \leq n \\
q-n & q>n
\end{array} ; \beta_{q}= \begin{cases}2 & q \leq n \\
1 & q>n .\end{cases}\right.
$$


Integration of Equation (4.22) yields

$$
\begin{aligned}
& \underline{Z}(\infty)=\underline{Z}\left(r_{t}\right)+\int_{r_{t}}^{\infty} \underline{M}(r) \cdot \underline{Z}(r) d r \\
& \underline{Z}(r)=\underline{Z}\left(r_{t}\right)+\int_{r_{t}}^{r} \underline{M}(x) \cdot \underline{Z}(x) d x
\end{aligned}
$$

which may be combined to yield

$$
\underline{Z}(\infty)=\left[\underline{I}+\int_{r_{t}}^{\infty} \underline{M}(r) d r\right] \cdot \underline{Z}\left(r_{t}\right)+\int_{r_{t}}^{\infty} \underline{M}(r) \cdot \int_{r_{t}}^{r} \underline{M}(x) \cdot \underline{Z}(x) d x d r .
$$

If the last term in Equation (4.23) is neglected, 26,27 we obtain the simple projection equation

$$
\begin{aligned}
& \underline{Z}(\infty)=\underline{S}\left(\infty, r_{t}\right) \cdot \underline{Z}\left(r_{t}\right) \\
& \underline{s}\left(\infty, r_{t}\right)=\underline{I}+\int_{r_{t}}^{\infty} \underline{M}(r) d r
\end{aligned}
$$

where the reactance matrix projection equation is obtained from Equations $(4.7),(4.8)$, and $(4.24)$

$$
\underline{R}(\infty)=\left[\underline{S}^{(1,1)} \cdot \underline{R}\left(r_{t}\right)+S^{(1,2)}\right] \cdot\left[S^{(2,1)} \cdot \underline{R}\left(r_{t}\right)+\underline{S}(2,2)\right]^{-1}
$$

with 
$\underline{s}=\left[\begin{array}{ll}\underline{s}^{(1,1)} & s^{(1,2)} \\ \underline{s}^{(2,1)} & s^{(2,2)}\end{array}\right]$

and $\underline{R}\left(r_{t}\right)=\underline{H}^{(1)}\left(r_{t}\right) \cdot \underline{H}^{(2)}\left(r_{t}\right)^{-1}$.

If the asymptotic form of the direct potential is substituted into Equation (4.24), the resulting matrix $\underline{s}$ may be evaluated in terms of the standard incomplete sine and cosine functions. Knirk ${ }^{15}$ has introduced the projection technique in more detail.

From the reactance matrix projection, Equation (4.25), we see that the corrections applied to $R_{1 j}\left(r_{t}\right)$ are asymmetric for $1 \neq j$. Therefore, for some scattering problems, the long-range behavior of the direct potential especially near threshold may cause the projection procedure to be inaccurate if the value chosen for $r_{t}$ is too small. It may be necessary to choose a larger value for $r_{t}$ so that the symetry and convergence of the reactance matrix are acceptable. 


\section{CHAPTER II}

\section{APPLICATION}

Application to a Model Problem

An untested method is often initially applied to a simplified problem in an attempt to judge its effectiveness without encountering any undue numerical complications. Moreover, after completing the calculation, the researcher is hopefully able to estimate the degree of success that he can expect when applying the method to the more complicated equations that arise in the description of electron-atom collisions. However, in order to retain some elements of realism, the model problem should mathematically imulate the main features of the electron-atom scattering equations.

The formalism discussed in Chapter I 18 inftially applied to a model two-channel problem defined by the coupled equations

$$
\begin{aligned}
{\left[\frac{d^{2}}{d r^{2}}-\frac{l_{1}\left(\ell_{1}+1\right)}{r^{2}}+k_{i}^{2}\right] F_{1 j}(r) } & =\sum_{n} v_{1 n}(r) F_{n j}(r)+2 Y_{0}\left(P_{1}, F_{1 j} ; r\right) P_{1}(r) \\
& +M_{1 j} P_{i}(r)
\end{aligned}
$$

where

$$
\begin{aligned}
& v_{11}(r)=-2\left(1+\frac{1}{r}\right) e^{-2 r} \\
& v_{22}(r)=\frac{24}{3}-\left(\frac{1}{4} r^{2}+\frac{3}{2} r+\frac{11}{2}+\frac{14}{r}+\frac{24}{r^{2}}+\frac{24}{r^{3}}\right) e^{-r}
\end{aligned}
$$




$$
\begin{aligned}
& v_{12}(r)=v_{21}(r)=-\frac{2}{3}\left[\frac{256}{81 r^{2}}-e^{-3 r / 2}\left(\frac{4}{3} r+\frac{32}{9}+\frac{128}{27 r}+\frac{256}{81 r^{2}}\right)\right] \\
& \ell_{1}=0, \ell_{2}=1 .
\end{aligned}
$$

The level splitting is chosen to be 0.75 Ry so that $k_{1}^{2}=k_{2}^{2}+0.75$. This model is an arbitrarily truncated version of the e-H scattering equations using a 18-2p close coupling approximation so that the model still contains (1) long-range off-diagonal dipole coupling; (11) one exchange term in each channel; and (111) one orthogonality term in each channel.

The unnormalized solution to Equation (4.27) is

$$
\begin{aligned}
& \text { ل) }_{1 j}(r)=\delta_{1 j} G_{i}^{(1)}\left(k_{i} r\right)+\int_{0}^{r} d x G_{i}^{(2,1)}(r \mid x)\left\{\sum_{n} v_{i n}(x) d h_{n j}(x)\right. \\
& \left.\left.+2 P_{i}(x) \int_{0}^{x} \Gamma \frac{1}{x}-\frac{1}{y}\right] P_{i}(y) d / 1 j(y) d y\right\}+\left(2 C_{1 j}+M_{i j}\right) \\
& x \int_{0}^{r} d x G_{1}^{(2,1)}(r \mid x) P_{1}(x) \\
& \left.{ }^{\prime \prime}\right)_{i j}^{(0)}(r)+\sum_{m} \mho_{i m}^{(1)} c_{m j}+\sum_{m} \|_{i m}^{(2)} M_{m j}
\end{aligned}
$$

where the auxiliary functions are defined as 


$$
\begin{aligned}
& \|_{i j}^{(n)}(r)=\delta_{\alpha 0} \delta{ }_{i j} G_{i}^{(1)}\left(k_{1} r\right)+\int_{0}^{r} d x G_{i}^{(2,1)}(r \mid x)\left\{\sum_{n} v_{1 n}(x)\right. \\
& \left.x v_{n j}^{(x)}(x)+2 p_{i}(x) \int_{0}^{x} d y\left[\frac{1}{x}-\frac{1}{y}\right] p_{i}(y) h_{i j}^{(w)}(y)\right\} \\
& +\delta_{1 j}\left(2 \delta_{\alpha 1}+\delta_{\alpha 2}\right) \int_{0}^{r} d x G_{1}^{(2,1)}(r \mid x) P_{1}(x)
\end{aligned}
$$

Notice that the inhomogeneous terms for $\alpha=1$ and $n=2$.differ only by a factor of two so that their auxiliary functions are related a 8

$$
\psi_{i j}^{(1)}(r)=2 \psi \psi_{i j}^{(2)}(r) .
$$

The exchenge constant 18

$$
\begin{aligned}
c_{i j} & =\int_{0}^{\infty} P_{i}(y) \psi_{i j}(y) y^{-1} d y \\
& =c_{i j}^{(0)}+\sum_{m} c_{i m}^{(1)} C_{m j}+\sum_{m} c_{i m}^{(2)} M_{m j}
\end{aligned}
$$

with

$$
c_{i j}^{(\alpha)}=\int_{0}^{\infty} P_{1}(y) \psi_{1 j}^{(\alpha)}(y) y^{-1} d y
$$

and the orthogonality constant is determined by 


$$
0=M_{i j}^{(0)}+\sum_{m} M_{i m}^{(1)} C_{m j}+\sum_{m} M_{i m}^{(2)} M_{m j}
$$

with

$$
M_{i j}^{(y)}=\int_{0}^{\infty} P_{i}(y) \psi_{i j}^{(\alpha)}(y) d y
$$

We solve Equation (4.27) by the NIEM method as follows:

(1) Obtain the auxiliary functions given in Equation (4.29) by using a convenient integration scheme. The power of the method comes from the fact that, as pointed out by Sams and Kour $1,{ }^{10}$ the solution at a given value of $r$ depends only a previously calculated values of $r$. For example, in Equation (4.29) we replace the integrals by quadrature sums,

$$
\begin{aligned}
& \|_{i j}^{(\alpha)}\left(r_{m}\right)=\delta_{\alpha 0^{\delta}} G_{i j}^{(1)}\left(k_{1} r_{m}\right)+\sum_{k=1}^{m} \omega_{k} G_{i}^{(2,1)}\left(r_{m} \mid r_{k}\right) \\
& \underset{n}{x\left\{\sum_{1 n}\right.} v_{k}\left(r_{k}\right) \psi_{n j}^{(N)}\left(r_{k}\right)+2 P_{i}\left(r_{k}\right) \underset{l=1}{k} \omega_{l}\left(r_{k}^{-1}-r_{l}^{-1}\right) \\
& \left.X P_{1}\left(r_{\ell}\right) \psi_{1 j}^{(\alpha)}\left(r_{\ell}\right)+\delta_{1 j}\left(2 \delta_{\alpha 1}+2 \delta_{\alpha^{2}}\right) P_{1}\left(r_{k}\right)\right\},
\end{aligned}
$$

where $w_{k}$ are the weights of the quadrature scheme. Now we have that there is zero contribution to the summations over $k$ and 0 . from the terms $k=m$ and $\ell=k$, respectively. 
Thus, the right-hand-side of this equation does not involve the unknown functions at $r_{m}$. However, this simplification does not directly occur with Numerov; rather, atrix inversion is required to express the solution at alven value of $r$ in terms of only previously calculated values of $r$. Since the matrix to be inverted at each grid point $r_{m}$ is of the same order as the solution matrix, it is apparent that a considerable amount of computation time can be consumed in performing all the matrix inversions, especially when a large number of channels 18 involved. (11) Solve for the constants $C_{m j}$ and $M_{m j}$. Since the constants $C_{i j}^{(w)}$ and $M_{i j}^{(\alpha)}$ are determined in step (i), $C_{m j}$ and $M_{m j}$ are obtained by solving the set of simultaneous equations given in Equation (4.31) and (4.32). (iii) Compute the reactance matrix $\underline{R}\left(r_{t}\right)$ and step out to $\underline{R}$. From the information gained in steps (1) and (11), the unnormalized solution $\downarrow(r)$ given in Equation $(4.27)$ can be computed at some transformation point $r_{t}$. The matrix $\underline{R}\left(r_{t}\right)$ is then computed from Equation (4.26) using $\underline{H}^{(1)}\left(r_{t}\right)$ and $\underline{H}^{(2)}\left(r_{t}\right)$ of Equation (4.7), and this matrix is projected to its asymptotic value using Equation (4.25).

We use the trapezoldal rule (TR) with a variable step size to integrate the equations. The simplicity of the TR makes its application to the equations a simple task. There 
is really no significant advantage in using another rule such as Simpson's rule (SR). In general, a significant advantage would be to apply SR with a larger step size and, at the same time, obtain results with at least the same accuracy of the TR, thus allowing a savings in computation time. However, we may be required to use a small step size if there are any tight loops in the solutions $\psi_{i j}^{(\alpha)}(x)$ for values of $r$ near the origin. Such tight loops are characteristic of the excited state wave functions in electron scattering by atomic oxygen. In order to avoid missing the tight loops, small step size must be used even with SR. However, the use of the TR with this step size also ylelds acceptable results. Of course, if no tight loops are present, SR would be preferred over the TR.

Furthermore, since integral equation techniques are inherently stable, we find that a relatively large step size may be used during a calculation. As we integrate the solutions $\psi_{1 j}^{(w)}(r)$ from the origin outwards, we may progressively increase the step size while maintaining a desired accuracy in the results. For example, we may start the integration with a step size of $0.02 a_{0}$ and double it at values of $r$ equal to $0.1 a_{0}, 0.5 a_{0}, 1.0 a_{0}$ and $5.0 a_{0}$. Thus, for values of $r$ greater than $5.0 a_{0}$, we are using a step size of $0.32 a_{0}$. This step size is quite large and will enable us to quickly reach the transformation point $r_{t}$ from 5.0 $a_{0}$ using the 'IR. To say that 
an even larger step size could be used for SR is not entirely correct. If too large a step size is chosen, the oscillatory behavior of the solutions could be overlooked. This 18 especially true when the channel energy la large and, thus, the solution is rapidly oscillating. With these observations in mind, we consider the use of the TR adequate.

Table I gives reactance matrix elements that agree with the numerical standard (Numerov) within $0.2 \%$. Two significant conments should be noted. First, the NIEM was at least a factor of 2 faster In obtaining the reactance matrix elements than Numerov for this two-channel problem. Second, at least $80 \%$ of the computation time is involved in integrating the auxiliary functions and in solving for the constants. The generation of the potentials and of the Green's functions, and the application of the projection procedure all consume little time.

We obtain the transformation point $r_{t}$ using a sequential projection procedure. The first projection point $r_{1}$, where we profect the reactance matrix out to an asymptotic value, is chosen to epproximate the point at which all the potentials, except the direct potential, no longer contribute. For our model problem, the point $r_{1}$ is chosen to approximate the point where the exchange potential terms no longer contribute. 
The second projection point $r_{2}$ is the point where the effect of the direct potential is somewhat small, and usually should he at least a distance of $10 \varepsilon_{0}$ from $r_{1}$. If the two projected reactance matrices from $r_{1}$ and $r_{2}$ agree to within our accepted accuracy of $0.1 \%$, we conclude that either $r_{1}$ or $r_{2}$ is the transformation point $r_{t}$. On the other hand, if the two profected reactance matrices do not agree, we must continue the process by choosing sequentially larger projection points until a transformation point is obtained. If we choose $r_{1}=30 a_{0}$ and $r_{2}=45 a_{0}$ for our model problem, we find that the projected reactance matrices agree to within $0.1 \%$ for most energies. However, Smith et al. have shown that for energies near a threshold, the direct potential is more extended. For this resson, we found it necessery to use a value of $r_{t}=600_{0}$ at energies of 0.749 and 0.751 . 
TABLE I

Reactance Matrix Elements at Energies Below and Above Threshold. Column (NIEM) Presents Results Calculated Using the NIEM; (STANDARD) Lists the Values Obtained Using NUMEROV

E (Ry)

0.1

0.2

0.5

0.7

0.749

0.751

$\mathbf{R}_{11}$

$\mathbf{R}_{12}$

$\mathbf{R}_{22}$

0.80

$\mathbf{R}_{11}$

$\mathrm{R}_{12}$

$\mathrm{R}_{22}$

$1.0 \quad R_{11}$

$\mathbf{R}_{12}$

$\mathrm{R}_{22}$

1.5
NIEM

$-1.471$

$-3.937$

3.287

1.870

1.718

1.713

0.0071

$-0.166$

1.572

0.240

$-2.738$

1.273

$-0.193$

0.686

0.891

$-0.220$

0.440
STANDARD

$-1.471$

$-3.935$

3.288

1.871

1.718

1.713

0.0071

$-0.166$

1.572

0.240

$-2.740$

1.273

$-0.193$

0.686

0.891

$-0.220$

0.440 
Application to Electron-Hydrogen Scattering

Due to the simplicity of the e-H scattering equations, untested techniques are often applied to them. However, the strong degenerate dipole coupling between the 28 and $2 p$ channels presents a formidable test ${ }^{7}$ of the reliability of a technique. In addition, there exists a series of resonances below the $n=2$ threshold that can be used to judge the ability of a method to locate resonances. Therefore, we have chosen the e-H scattering problem as a physical test of the applicability of the NIEM.

The e-H scattering equations ${ }^{28}$ in the $18-28-2 p$ close coupling approximation for $L=0$ consist of three coupled integro-differential equations that can be cast into three coupled integral equations of the Volterra type. Application of the NIEM is a simple and straightforward procedure.

The three coupled equations are solved using integral equation theory from zero out to some transformation point $r_{t}$ where the reactance matrix is projected out to its asymptotic value by using atricant technique. This value of $r_{t}$ is at least the point at which the integrals involving the exchange and orthogonality terms have converged. Thus, any further contribution to the solution comes only from the direct potential. We use the trapezoldal rule, with a variable step size, to integrate the coupled equations. An Important 
advantage of suing the method comes from the fact that, as pointed out by Sams and Kouri, ${ }^{10}$ the solution at aiven value of $r$ depends only on previously calculated values of $r$.

A comparison of our results with those of Burke et al. 29 indicated that there was not agreement at all the published energies. Their results were obtained with an older version of Numerov where the asymptotic expansion method of Burke and Schey ${ }^{17}$ was not used in obtaining the reactance matrix. Thus, we solved the three coupled integro-differential equations using Numerov to obtain solutions as a standard against which results from the NIEM may be judged.

Table II presents reactance matrix elements for energies above the $n=2$ threshold. When applying the NIEM, the results quoted are computed using a transformation value of $500 a_{0}$ with exchange and orthogonality terms omitted for values of $r>300_{0}$. Since the dipole coupling between the 28 and $2 p$ degenerate channels is large for energies just above threshold, ${ }^{7}$ in this region larger transformation point $r_{t}$ should be used for convergence purposes. For example, at an energy of 0.76 Ry., an asymmetry within $1 \%$ exists in the reactance matrix calculated with $r_{t}=500 a_{0}$. However, the partial wave cross sections are more reliable than the reactance matrix elements at this enersy due to their more rapid convergence. ${ }^{7,30}$ Row (a) present s 
the NIEM results, while row (b) contains the results of Burke et $a^{29}$ Whenever any elements between the two rows disagree by more than 18 , we insert row (c) which presents results of Numerov. We note that some of the reactance matrix elements of Burke and coworkers contained In Table II are in error by $A$ much $a 8$ 10\%. Table III containe partial wave cross sections only for the cases where row (c) is needed. A1though there is up to $10 \%$ disagreement in the reactance matrix elements, the cross sections agree to within $2 \%$.

The phase shifts for energies below threshold agree to within $0.2 \%$ with the results of Burke et $a 1 .^{31}$ The results are computed by integrating the scattering equations out to $30 a_{0}$ with exchange and orthogonality terms being retained to this point. We further calculated the energy and width of the first scattering resonance below the $n=2$ threshold. The energy and width are $E_{r}=9.573$ and $\Gamma=0.0545 \mathrm{eV}$ from the NIEM and $E_{r}=9.575$ and $\Gamma=0.0543 \mathrm{eV}$ from Burke et al. 32 Just below threshold, the closed channel components of the scattering wave function are extended; thus, in order to obtain accurate phase shifts, it is necessary to integrate the equations out to $100 a_{0}$, while exchange and orthogonality terms are st111 dropped at $300_{0}$.

Knirk, et al. 16 have also extended the work of Sams and Kouri $i^{10}$ and applied their version of a NIEM only to open channel scattering of electrons by atomic hydrogen. However, they were 
unable toiobtain reliable results for singlet and triplet S-wave cross sections at an energy of $2.25 \mathrm{Ry}$. The shortcoming can be avoided by separating the exchange potential terms in the manner given in Equations (4.13) - (4.15). 


\section{TABLE II}

Reactance Matrix Elements at Energies Above the $n=2$ Threshold.

Row (a) Present Results; (b) Ref. [29] 1180 in the 18-28-2p Approximation; (c) Numerov.

\begin{tabular}{llllllll}
\hline \multirow{2}{*}{$\mathbf{k}^{2}=0.76$} & $\mathrm{R}_{11}$ & $\mathrm{R}_{12}$ & $\mathrm{R}_{13}$ & $\mathrm{R}_{22}$ & $\mathrm{R}_{23}$ & $\mathrm{R}_{33}$ \\
& a) & -0.5438 & 3.047 & -5.891 & -7.274 & 13.17 & -23.19 \\
Singlet & b) &.-- & --- & --- & --- &.-- & --- \\
& c) & -0.5480 & 3.055 & -5.909 & -7.286 & 13.19 & -23.22 \\
& & & & & & & \\
& a) & 36.61 & 0.5491 & -1.053 & -1.276 & 1.510 & -0.5590 \\
Triplet & b) & --- & --- & --- & --- & --- & --- \\
& c) & 37.23 & 0.5560 & -1.076 & -1.276 & 1.510 & -0.5573
\end{tabular}


TABLE II

(continued)

\begin{tabular}{|c|c|c|c|c|c|c|c|}
\hline & & $\mathrm{R}_{11}$ & $\mathrm{R}_{12}$ & $\mathrm{R}_{13}$ & $\mathrm{R}_{22}$ & $\mathrm{R}_{23}$ & $\mathrm{R}_{33}$ \\
\hline & a) & 0.9330 & -0.2851 & 0.5295 & -0.6427 & 0.1377 & 1.923 \\
\hline \multirow[t]{3}{*}{ Singlet } & b) & --- & -- & -- & -- & -- & --- \\
\hline & c) & 0.9339 & -0.2853 & 0.5303 & -0.6426 & 0.1376 & 1.923 \\
\hline & a) & 25.23 & -0.7765 & 1.107 & 0.3852 & -1.426 & 3.955 \\
\hline \multirow[t]{2}{*}{ Iriplet } & b) & -- & -- & $\cdots$ & --- & -- & $--\cdot$ \\
\hline & c) & 25.50 & -0.7880 & 1.122 & 0.3864 & -1.427 & 3.957 \\
\hline
\end{tabular}


TABLE II

\section{(Continued)}

\begin{tabular}{|c|c|c|c|c|c|c|c|}
\hline & & $\mathbf{R}_{11}$ & $\mathrm{R}_{12}$ & $\mathbf{R}_{13}$ & $R_{22}$ & $R_{23}$ & $R_{33}$ \\
\hline & a) & 0.8232 & -0.2759 & 0.5897 & -1.101 & 1.021 & 0.5887 \\
\hline \multirow[t]{2}{*}{ Singlet } & b) & 0.8189 & -0.2763 & 0.5863 & -1.099 & 1.021 & 0.5841 \\
\hline & a) & 16.90 & -0.3859 & 0.3943 & -0.6846 & -0.0108 & 1.403 \\
\hline \multirow[t]{2}{*}{ Triplet } & b) & 16.66 & -0.3650 & 0.3940 & -0.6858 & -0.0094 & 1.402 \\
\hline & c) & 17.01 & -0.3876 & 0.3978 & -0.6845 & -0.0106 & 1.403 \\
\hline
\end{tabular}


TABLE II

(continued)

$\begin{array}{llllll}R_{11} & R_{12} & R_{13} & R_{22} & R_{23} & R_{33}\end{array}$

$$
k^{2}=1.00
$$

$\begin{array}{rllllllr} & \text { a) } & 0.2926 & -0.0043 & 1.698 & 0.0198 & 1.475 & -5.509 \\ \text { Singlet } & \text { b) } & 0.2891 & -0.0059 & 1.701 & 0.0184 & 1.478 & -5.524 \\ & \text { c) } & 0.2930 & -0.0043 & 1.700 & 0.0198 & 1.475 & -5.511\end{array}$

$\begin{array}{lllllll}\text { a) } & 7.196 & 4.325 & -2.400 & 15.57 & -9.491 & 5.658 \\ \text { b) } & 7.205 & 4.553 & -2.561 & 16.78 & -10.32 & 6.219 \\ \text { c) } & 7.216 & 4.327 & -2.401 & 15.51 & -9.456 & 5.637\end{array}$


TABLE II

(continued)

\begin{tabular}{llllllll}
\hline & & $R_{11}$ & $R_{12}$ & $R_{13}$ & $R_{22}$ & $R_{23}$ & $R_{33}$ \\
$k^{2}=1.21$ & & & & & & & \\
& a) & 0.1491 & 0.5052 & 1.621 & 0.1560 & -0.5206 & -5.868 \\
Singlet & b) & 0.1443 & 0.5051 & 1.632 & 0.1545 & -0.5242 & -5.914 \\
& c) & 0.1500 & 0.5057 & 1.623 & 0.1558 & -0.5215 & -5.871 \\
& & & & & & & \\
& & & & & & & \\
Triplet & b) 3.889 & 0.5389 & -0.1942 & 1.056 & -1.492 & 0.7018
\end{tabular}


TABLE II

(continued)

\begin{tabular}{|c|c|c|c|c|c|c|c|}
\hline \multirow{2}{*}{$k^{2}=1.4$} & & $\mathrm{R}_{11}$ & $R_{12}$ & $\mathrm{R}_{13}$ & $\mathrm{R}_{22}$ & $\mathrm{R}_{23}$ & $\mathrm{R}_{33}$ \\
\hline & a) & 0.2659 & 0.7380 & 0.8255 & -0.4220 & -1.522 & -2.974 \\
\hline \multirow[t]{3}{*}{ Singlet } & b) & 0.2624 & 0.7389 & 0.8292 & -0.4261 & -1.529 & -2.989 \\
\hline & c) & 0.2676 & 0.7383 & 0.8256 & -0.4231 & -1.524 & -2.975 \\
\hline & a) & 2.864 & 0.4289 & -0.0880 & 0.0763 & -1.327 & 0.3798 \\
\hline \multirow[t]{2}{*}{ Triplet } & b) & 2.859 & 0.4270 & -0.0879 & 0.0746 & -1.327 & 0.3798 \\
\hline & c) & 2.863 & 0.4290 & -0.0879 & 0.0754 & -1.326 & 0.3798 \\
\hline
\end{tabular}


TABLE II

(continued)

\begin{tabular}{llllllll}
\hline & & $R_{11}$ & $R_{12}$ & $R_{13}$ & $R_{22}$ & $R_{23}$ & $R_{33}$ \\
$k^{2}=2.25$ & & & & & & \\
& a) & 0.1244 & 1.401 & 0.7454 & -3.720 & -3.040 & -1.517 \\
Singlet & b) & 0.1199 & 1.408 & 0.7505 & -3.744 & -3.055 & -1.527 \\
& c) & 0.1234 & 1.404 & 0.7472 & -3.733 & -3.047 & -1.522 \\
& & & & & & & \\
& & & & & & & \\
Triplet & b) 1.628 & 0.5991 & 0.1035 & -2.457 & -1.994 & -0.1209
\end{tabular}




\section{TABLE II}

(continued)

$$
\mathrm{R}_{11}
$$

$$
k^{2}=4.00
$$

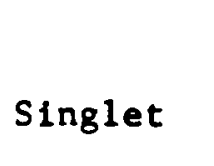
a) 0.8502

$-1.187$

$-0.5931$

$-1.167$

$-1.186$

$-0.5836$

$-0.5927$

c) 0.8503

a) 1.231

$-0.9270$

$-0.4796$

$-0.9043$

$-0.4699$

$\begin{array}{ll}-0.9270 & -0.4798\end{array}$

$\begin{array}{ll}-0.9270 & -0.4798\end{array}$

c) 1.231

7.337

2.455

1.720

7.212

2.406

1.700

7.341

2.457

1.720 
TABLE III

Partial Wave Cross Sections at Energles Above the $n=2$ Threshold. Row (a) Present Results; (b) Ref. [29] and Ref. [31].

$k^{2}=0.81$
a) $\quad 3.687$
0.0013
0.0007
16.22

Triplet
b) $\quad 3.687$
0.0012
0.0007
16.24

$k^{2}=1.00$
a) 0.2647
0.0768
0.0360
0.2883

Singlet
b) $\quad 0.2635$
0.0766
0.0360
0.2876

a) $\quad 2.907$

0.0037

0.0033

6.310

Triplet
b) $\mathbf{2 . 9 0 6}$
0.0036
0.0033
6.251

$k^{2}=1.21$
a) 0.1731
0.0589
0.0359
0.0659

Singlet
b)
0.1722
0.0588
0.0359
0.0654 
$k^{2}=1.44$
a) 0.1278
0.0381
0.0343
0.1670

Singlet
b) 0.1269
0.0380
0.0343
0.1665
•) 1.827
0.0055
0.0096
1.718

Triplet
b) $\quad 1.827$
0.0055
0.0095
1.719

$k^{2}=2.25$
.) 0.0842
0.0123
0.0172
0.3737

Singlet
b) 0.0836
0.0123
0.0171
0.3737

$k^{2}=4.00$
a) 0.0589
0.0049
0.0035
0.2415

Singlet
b) $\quad 0.0579$
0.0049
0.0035
0.2412
a) 0.4002
0.0030
0.0052
0.7894

Triplet
b) 0.3970
0.0030
0.0052
0.7894 
Application to Electron-0xygen Scattering

As a final test for the entire NIEM formalism let us consider the scattering of low-energy electrons by atomic oxygen. The cross sections are obtained using a four state close coupling approximation where the total wave function for the system is expanded in terms of real states of atomic oxygen. The only atomic states retained in the expansion are those of the ground state configuration $1 s^{2} 2 s^{2} 2 p^{4}\left({ }^{3} P,{ }^{1},{ }^{1} S\right)$ and the excited state configuration $\left.1 s^{2} 2 s^{2} 2 p^{3} 3 s^{3} s^{0}\right)$. We choose only to investigate $P$-wave scattering because of 1 ts dominant contribution to the total elastic ${ }^{3} \mathrm{P} \rightarrow^{3} \mathrm{P}$ cross section (see Table IV). To investigate P-wave scattering, let us briefly review the channel designations, orthogonalization requirements, and the electron capture states used in solving Eqation (4.1) Consider the channel designation chart:

\begin{tabular}{ccccccc}
$\mathrm{L}$ & $\mathrm{S}$ & $\pi$ & ${ }^{3}$ & $1_{\mathrm{D}}$ & $\mathbf{1}_{\mathrm{S}}$ & $3 \mathrm{e}^{3} \mathrm{~S}^{0}$ \\
\hline 1 & $1 / 2$ & 0 & 1 & 1,3 & 1 & 1 \\
1 & $1 / 2$ & $\mathrm{e}$ & 0,2 & 2 & \\
1 & $3 / 2$ & 0 & 1 & & \\
1 & $3 / 2$ & e & 0,2 & &
\end{tabular}

where I, and $S$ are the total orbital and spln angular momenta, $\pi$ is the parity either even (e) or odd (0), the overall 
coupling of the atomic states is ${ }^{2 S+1} L^{\pi}=\left({ }^{3} P,{ }^{1} D,{ }^{1} S,{ }^{3} S^{0}\right)$, and the allowed incident angular momenta $l$ are obtained using the triangle sum rule. Since we want to investigate the effect of including the $3 s^{3} s^{0}$ state, we need only consider the ${ }^{2} P$ and ${ }^{4} P$ cases. For an example of channel designation consider the ${ }^{2} \mathrm{p}$ case where channel one is characterized by $\left({ }^{3} P, \ell=0\right)$, two by $\left({ }^{3} P, \ell=2\right)$, three by $\left({ }^{1} D, l=2\right)$, and four by $\left(38^{3} s^{0}, l=1\right)$. From the solution of the appropriate coupled equations, we obtain either the ${ }^{2} P$ or ${ }^{4} p$ contributions to the cross sections among the included atomic states. Next, the only electron capture state that is needed for the ${ }^{2} \mathrm{P}$ cose is $\Phi\left(1 s^{2} 2 s^{2} 2 p^{4} 38 ;{ }^{2} P\right)$, and for the ${ }^{4} P$ case is $\Phi\left(1 s^{2} 2 s^{2} 2 p^{4} 38 ;{ }^{4} P\right)$. Finally, the orthogonalization requirements for both cases are: (1) channels in which $l=0$ are radially orthogonalized to the $18,28,38$, orbitals; (2) channel in which $\ell=1$ is radially orthogonslized to the $2 p$ orbital.

Table V presents the ${ }^{4} \mathrm{P}$ contributions to the elastic ${ }^{3} \mathrm{P} \rightarrow{ }^{3} \mathrm{P}$ cross section. Column (a) contains the NIEM results where we use a transformation point of $r_{t}=57 a_{0}$ with exchange, correlation and orthogonality terms omftted for values of $r$ greater than $25 a_{0}$. Column (b) contains the results ${ }^{33}$ obtained with another numerical procedure (Numerov) described earlier. Column (c) contains previously published results ${ }^{34}$ using close coupling method with only the three ground state configuration terms retained in the expansion of the total 
wave function. First, we see that the NIEM and Numerov agree to within 0.1\%. Second, we note that the difference between the results of the three and four state expansions is small at high energies and as large as $10 \%$ at low energies. Also not that the NIEM was at least a factor of 4 faster in obtaining the final results than Numerov for this 3-channel problem.

Table VI presents ${ }^{2} \mathrm{P}$ contributions to elastic and inelestic cross sections. Rows (a), (b), and (c) are of the same origin and Columns (a), (b), and (c) of Table V. First, we see that the NIEM and Numerov agree to within $0.3 \%$. Second, we note that the difference between the results of the three and four state expansion is small at high energies and as large as $25 \%$ at lower energies. Also note that the NIEM was at least a factor of 6 faster in obtaining the final results than Numerov for this 4-channel problem.

The large drop in the $P$-wave cross eections at low energies using a 4 state close coupling expansion is common to both tables and should be expected. First, we note the 3 state close coupling expansion of Smith et al. ${ }^{34}$ yielded total elastic ${ }^{3} \mathrm{P}^{3}{ }^{3} \mathrm{P}$ cross sections in disagreement with experimental cross sections of Sunshine et al. 35 by as much as $75 \%$ at low energles. They attributed their overestimate in the total cross section to the neglect of polarization. Temkin ${ }^{36}$ attempted to include this effect by using a polarized orbital method, 
1.e., a method that includes the reaction of the scattered electron back on the atom. His resulto are in falr agreement with the experimental results of Sunshine et al. ${ }^{35}$ Also, Neynaber et al. ${ }^{37}$ have measured the total elastic ${ }^{3} P \rightarrow{ }^{3}$ cross section for electrons scattered by atomic oxygen. Their results are about $30 \%$ smaller than the results of Sunshine et al. 35 Thus, the total elastic ${ }^{3} \mathrm{P}_{\rightarrow}{ }^{3} \mathrm{P}$ cross section using the 3 state close coupling expansion is even higher than their experimental cross section. However, our P-wave partial cross sections using a 4-8tate rather than 3-8tate close coupling expansion clearly indicate a substantial decrease in the total elastic ${ }^{3} \mathrm{P} \rightarrow{ }^{3} \mathrm{P}$ cross sections at lower energies (see Figure 1). It is also clear that the close coupling method using only real states is ineffective unless more atomic states are retained in the expansion for the total wave function. However, the convergence to the final results by adding more and more atomic states would be slow; thus, the procedure would not be feasible because of the large amount of computer time and storage requirements necessary to solve the large number of resulting coupled integro-differential equations. To avoid the poor convergence and still obtain results in fair agreement with experiment, we could supplement the close coupling expansion with pseudo-state orbitals (i.e., excited states that allow for more long-range polarization effects and short-range correlations to be included in the close coupling expansion). 
TABLE IV

Partial Wave Contributions to the Total Elastic ${ }^{3} \rightarrow^{3}{ }^{3}$ Cross Section $\left(\pi a_{0}^{2}\right)$ Using a 3-State Approximation

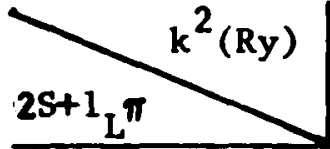

0.1

0.2

0.3

0.4

${ }^{2} s^{0}$

0.107

0.140

0.163

0.179

${ }^{4} s^{0}$

0.214

0.280

0.327

0.357

${ }^{2} \mathbf{P}$

3.271

2.918

2.613

2. 347

${ }^{2}{ }^{0}$

1.519

1.577

1.449

1.308

${ }^{4}$ P

4.324

4.116

3.891

3.664

${ }^{4} \mathrm{P}^{0}$

0.000

0.050

0.134

0.220

${ }^{2} \mathrm{D}$

0.009

0.008

0.007

0.006

${ }^{2} D^{0}$

0.166

0.361

0.521

0.642

${ }^{4} \mathrm{D}$

0.021

0.030

0.044

0.062

${ }_{11}^{40}$

0.209

0.453

0.669

0.843 
TABLE V

${ }^{4} P$ Contributions to the Elastic Cross Section $Q\left({ }^{3} P \rightarrow{ }^{3} P\right)$. Column

(a) Present Results; (b) Ref. [33]; (c) Ref. [34].

$\begin{array}{lrrr}E(\text { Ry }) & (a) & (b) & (c) \\ 0.10 & 3.946 & 3.949 & 4.324 \\ 0.15 & 3.986 & 3.988 & 4.222 \\ 0.20 & 3.961 & 3.962 & 4.116 \\ 0.25 & 3.905 & 3.905 & 4.005 \\ 0.30 & 3.824 & 3.824 & 3.891 \\ 0.40 & 3.628 & 3.628 & 3.664 \\ 0.50 & 3.420 & 3.419 & 3.441 \\ 0.60 & 3.214 & 3.214 & 3.226 \\ 0.80 & 2.820 & 2.819 & 2.832 \\ 1.00 & 2.484 & 2.484 & 2.489\end{array}$


TABLE VI

${ }^{2}$ p Contributions to the Elastic and Inelastic Cross Sections. Row (a) Present Results; (b) Ref. [33]; (c) Ref. [34]

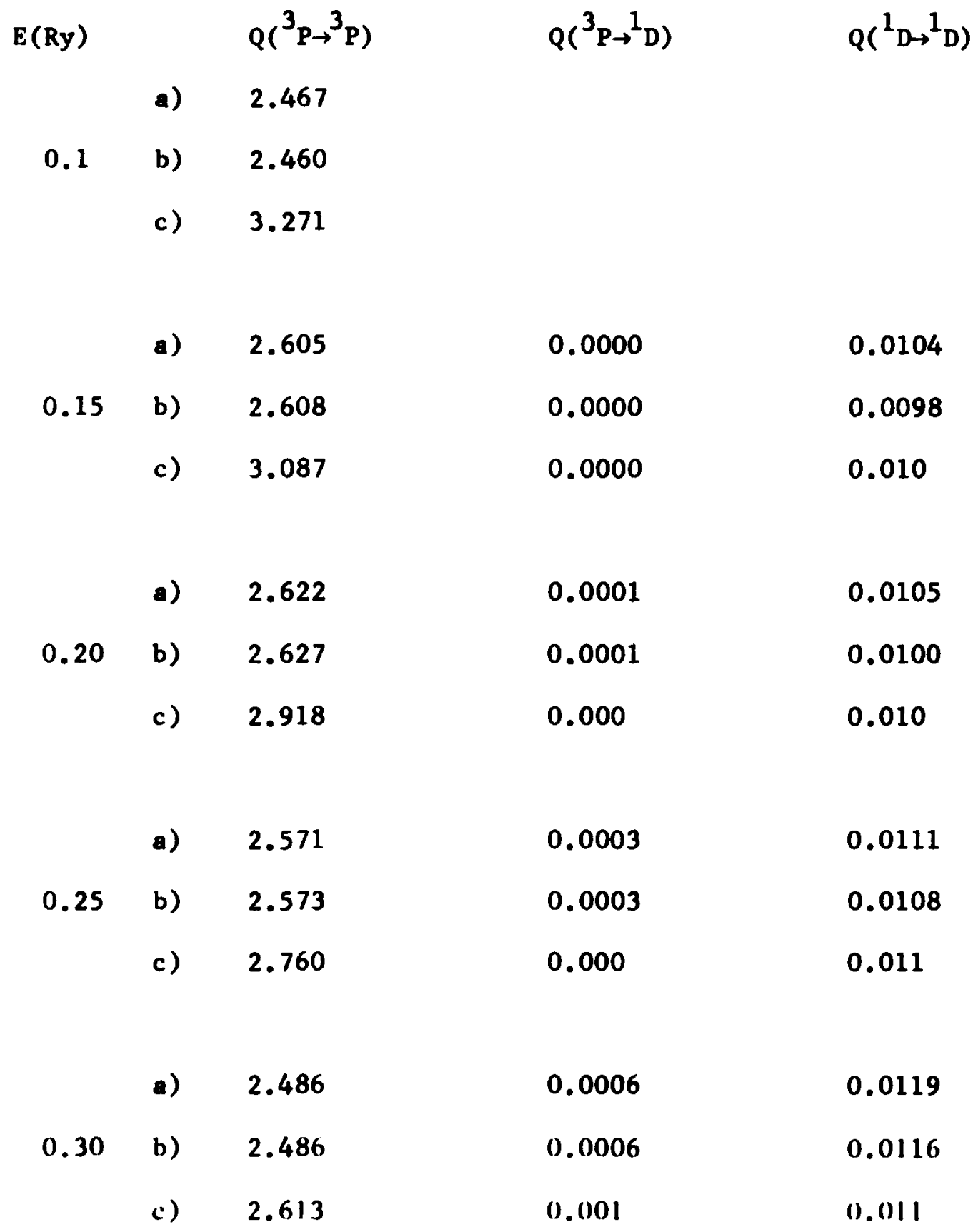


TABLE VI

(continued)

\begin{tabular}{|c|c|c|c|c|}
\hline$E(R y)$ & & $Q\left({ }^{3} P \rightarrow^{3} P\right)$ & $Q\left({ }^{3} P \rightarrow{ }^{1} D\right)$ & $Q\left({ }^{1} \mathrm{D} \rightarrow \mathrm{D}\right.$ \\
\hline \multirow{3}{*}{0.40} & a) & 2.281 & 0.0015 & 0.0140 \\
\hline & b) & 2.281 & 0.0015 & 0.0138 \\
\hline & c) & 2.347 & 0.001 & 0.014 \\
\hline \multirow{4}{*}{0.50} & a) & 2.075 & 0.0026 & 0.0167 \\
\hline & b) & 2.076 & 0.0026 & 0.0166 \\
\hline & c) & 2.116 & 0.003 & 0.016 \\
\hline & a) & 1.885 & 0.0039 & 0.0201 \\
\hline \multirow[t]{2}{*}{0.60} & b) & 1.885 & 0.0039 & 0.0200 \\
\hline & c) & 1.913 & 0.004 & 0.020 \\
\hline \multirow{3}{*}{0.80} & a) & 1.541 & 0.0066 & 0.0284 \\
\hline & b) & 1.541 & 0.0066 & 0.0284 \\
\hline & c) & 1.578 & 0.007 & 0.028 \\
\hline \multirow{3}{*}{1.00} & a) & 1.296 & 0.0094 & 0.0380 \\
\hline & b) & 1.296 & 0.0094 & 0.0380 \\
\hline & c) & 1.315 & 0.010 & 0.038 \\
\hline
\end{tabular}




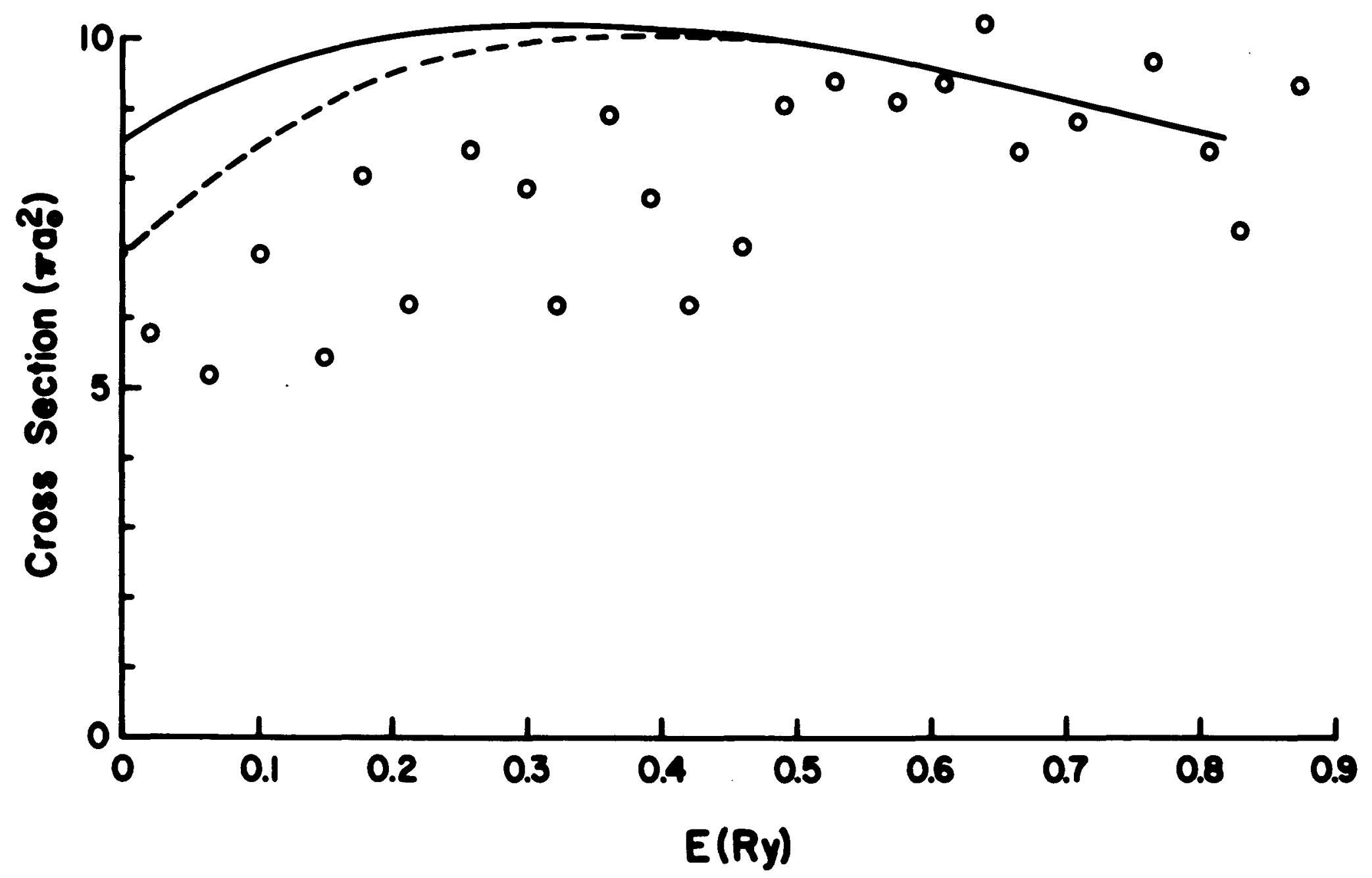

Figure I 


\section{CONCLUSION}

We have presented a simple computational procedure for solving a large number of coupled integro-differential equations which describe a multichannel scattering process. The complete effectiveness of the NIEM as compared to Numerov was demonstrated when applied to a two-channel model problem, electron scattering by atomic hydrogen, and electron scattering by atomic oxygen. (The testing of the method by applying it to selected problems was necessary since a method may not be completely computationally designed, i.e., even though a method solves a problem in principle, it may fail, in part, when first applied to a problem since it may not be able to successfully use already existing computational techniques).

The solution to the coupled equations and the associated boundary conditions of the scattering process is obtained from an unnormalized solution to the coupled equations by numerically forcing the open and closed asymptotic boundary conditions. The unnormalized solution can be obtained in the region where exchange, correlation, and orthogonality are important, by a simple technique analogous to the homogeneous and particular solutions of differential equation theory. We expand the unnormalized solution in terms of homogeneous and particular auxiliary functions that each satisfy their own separate integral equations. These auxiliary integral equations are 
solved from zero out to a transformation point where the unnormalized solution is obtained by recombining the auxillary functions. Now, the closed and open channel boundary conditions may be forced on the unnormalized solution. After computing the reactance matrix at the transformation point, it may be projected out to its asymptotic value using a matricant technique.

In closing, we see that there are two basic advantages in using the NIEM. First, due to the simplicity of the method, the programming required to solve a set of scattering equations is neither extensive nor involved. Second, the method offers a computational procedure with rellability, accuracy, and speed. The NIEM was significantly faster than Numerov in obtaining the reactance matrix elements for all cases examined; we consider its accuracy to be superior. 


\section{BI BLIOGRA PHY}

1. B. L. Moiseiwitsch and S. J. Smith, Reviews of Modern Physics 40, 255 (1968).

2. M. J. Conneely, L. Lipsky, K. Smith, P. G. Burke, and R. J. W. Henry, Comp. Phys. Comn. 1, 306 (1970).

3. P. G. Burke and H. M. Schey, Phys. Rev. A126, 147 (1962).

4. K. Smith, R. J. W. Henry, and P. G. Burke, Phys . Rev. A147, $21(1966)$

5. F. E. Harris, Phys. Rev. Letters 19, 173 (1967).

6. R. K. Nesbet, Phys. Rev. A175, 134 (1968); 179, 60 (1969).

7. E. R. Smith, R. S. Obero1, and R. J. W. Henry, J. Comput. Phys. 10, 53 (1972).

8. P. G. Burke, A. Hibbert, and W. D. Robb, J. Phys. B厽, 153 (1971).

9. J. J. Matese and R. J. W. Henry, Phys. Rev. AS, 222 (1972).

10. W. N. Sams and D. J. Kouri, J. Chem. Phys. 51, 4809 (1969).

11. W. N. Sams and D. J. Kouri, J. Chem. Phys. 51, 4815 (1969).

12. W. N. Sams and D. J. Kour1, J. Chem. Phys. 52, 4144 (1970).

13. W. N. Sams and D. J. Kouri, J. Chem. Phys. 53, 496 (1970).

14. R. G. Newton, J. Chem. Phys. 53, 1298 (1970).

15. D. L. Knirk, J. Chem. Phys. 57, 4782 (1972).

16. D. L. Knirk, E. F. Hayes and D. J. Kouri, J. Chem. Phys. 57, 4770 (1972). 
17. E. R. Smith and R. J. W. Henry, Phys. Rev. AI, 1585 (1973).

18. E. R. Smith and R. J. W. Henry, Phys. Rev. A (1973).

19. D. Secrest in "Methods in Computational Physics" Vo1. 10, Eds. B. Alder, S. Fernbach, and M. Rotenberg, (Academic Press, New York, 1971), p. 243.

20. L. D. Landau and E. M. Lifshitz, Quantum Mechanics, (New York: Pergamon Press, 1965), Pp. 101-104.

21. G. Arfken, Mathematical Methods for Physicists, (New York: Academic Press, 1970), pp. 748-752.

22. K. Smith and Morgan, Phys. Rev. A165, 110 (1968).

23. U. Fano, Phys. Rev. A140, A67 (1965).

24. M. Abramowtiz and I. A. Stegun, Handbook of Mathematical Functions (Dover Pub1., Inc., New York, 1965).

25. W. Eastes and D. Secrest, J. Chem. Phys. 56, 640 (1972).

26. Private communication from Dr. D. J. Kouri.

27. F. R. Gantmacher, The Theory of Matrices (Chelsea Pub. Co., New York, 1964).

28. I. C. Percival and M. J. Seaton, Proc. Camb. Phil. Soc. 53, 654 ( 1957 ).

29. P. G. Burke, H. M. Schey, and K. Smith, Phys. Rev. 129, 1258 (1963).

30. F. E. Harris and H. Michels, Phys. Rev. Letters 22, 1036 (1969).

31. P. G. Burke, D. F. Gallaher, and S. Geltmann, J. Phys. B2, 1142 (1969). 
32. P. G. Burke, S. Ormonde, and W. Whittaker, Proc. Phys. Soc. (London) 92, 319 (1967).

33. S. P. Rountree (private communication).

34. K. Smith, R. J. W. Henry, and P. G. Burke, Phys. Rev. A157, $51(1967)$.

35. G. Sunshine, B. B. Aubrey, and B. Bederson, Phys. Rev. 154, $1(1967)$.

36. A. Temkin, Phys. Rev. 107, 1004 ( 1957 ).

37. R. H. Neynaber, L. L. Marino, E. W. Rothe, and S. M. Truj1110, Phys. Rev. $\underline{123}, 148$ (1961). 
VITA

Ed Ray Smith was born In Alexandria, Louisiana, on March 24, 1948. He graduated from Sulphur High School in Sulphur, Louls Iana, in 1966. He received both his B.S. and his M.S. degrees in physics from Louisiana State University in 1971 and 1972, respectively. 
Candidate: $\quad$ Ed Ray Smith

Major Field: $\quad$ Phystcs

Title of Thesis: Noniterative Integral-Equation Approach to Scattering Problems Approved:

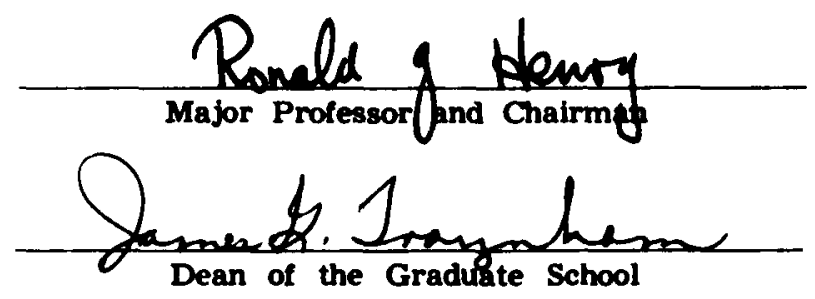

EXAMINING COMMITTEE:
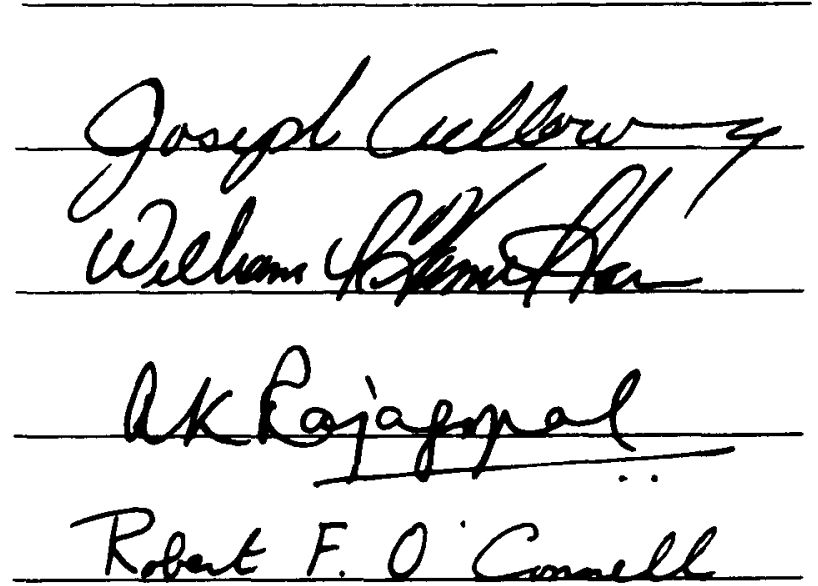

Date of Examination:

June 25, 1973 